\title{
A dynamic feedback mechanism with attitudinal consensus threshold for minimum adjustment cost in group decision making
}

\author{
Qi Sun, Jian Wu, Senior Member, IEEE, Francisco Chiclana, Hamido Fujita, Senior Member, IEEE, \\ and Enrique Herrera-Viedma, Fellow, IEEE
}

\begin{abstract}
This article presents a theoretical framework for a dynamic feedback mechanism in group decision making (GDM) by the implementation of an attitudinal consensus threshold (ACT) to generate recommendation advice for the identified inconsistent experts with the aim to increase consensus. The novelty of the approach resides in its ability to implement the ACT continuously, which allows the covering of all possible consensus states of the group from its minimum to maximum consensus degrees. Therefore, it can be flexibly applied to GDM problems with different consistency requirements. A sensitivity analysis method with visual simulation is proposed to support the checking of the numbers of experts involved in the feedback process and the minimum adjustment cost associated with the different ACT intervals. Experimental results show that an increase in the ACT value will lead to an increase in the number of experts and adjustment cost involved in the feedback process. Eventually, a numerical example is included to simulate the feedback process under various decision making scenarios with different ACT intervals.
\end{abstract}

Index Terms-Group decision making; Dynamic feedback mechanism; Attitudinal consensus threshold.

\section{INTRODUCTION}

I $\mathrm{N}$ group decision making (GDM) problems, a group of experts express their preference on a finite set of possible alternatives $X=\left\{x_{1}, \ldots, x_{n}\right\}$, which are fused into collective preferences before the application of a selection process to achieve a common agreed solution [1]-[4]. It is recognised that experts' background and knowledge differences may lead to conflict/inconsistency among them and be an obstacle in the achievement of group consensus [5]. Thus, before the aggregation stage, it would be beneficial that experts' preferences

Qi Sun and Jian Wu are with the School of Economics and Management, Shanghai Maritime University, Shanghai 201306, China (qisun1207@126.com; jyajian@163.com ) and with the Center for Artificial Intelligence and Decision Sciences, Shanghai Maritime University, Shanghai 201306, China.

Francisco Chiclana is with the Institute of Artificial Intelligence (IAI), Faculty of Computing, Engineering and Media, De Montfort University, Leicester, UK (chiclana@dmu.ac.uk) and with the Dept. of Computer Science and Artificial Intelligence University of Granada, Spain.

Hamido Fujita is with the Faculty of Information Technology, Ho Chi Minh City University of Technology (HUTECH), Ho Chi Minh City, Viet Nam and (h.fujita@hutech.edu.vn; HFujita-799@acm.org) with the Andalusian Research Institute in Data Science and Computational Intelligence, University of Granada, Granada, Spain and Iwate Prefectural University, Iwate, Japan.

Enrique Herrera-Viedma is the Andalusian Research Institute on Data Science and Computational Intelligence (DaSCI), University of Granada, Spain (viedma@decsai.ugr.es) and with the School of Business Administration, Southwestern University of Finance and Economics, Chengdu 610074, China. are close enough to guarantee the achievement of consensus [6]-[11]. In order to reduce inconsistency, researchers in the field of GDM have proposed a variety of consensus methods based on the implementation of a feedback mechanism [12][15]. These models are usually coordinated by a moderator responsible for supervising and guiding the experts in the overall feedback process, as well as for giving inconsistent experts, i.e. experts with consensus degrees below a group consensus threshold value, advice on how to modify their preferences [16]-[22]. It is noteworthy that the effectiveness of a feedback mechanism is affected by the group consensus threshold value used to identify the inconsistent experts. However, the threshold values of group consensus are different for different decision-making scenarios. So far, this has not been reported in the existent literature about feedback mechanism.

Therefore, one of the most important issues in reaching consensus regards how to select the appropriate threshold value in order to meet the different demands of multiple GDM organizations. In the aforementioned existent feedback mechanisms, a fixed consensus threshold value $\gamma$ to measure the level of group consensus is usually assumed based on the moderator's experience and knowledge [23]-[27]. Neither the feedback process number of inconsistent experts nor their associated adjustment cost can be known before the consensus threshold is determined. However, in practice, the consensus threshold may be different for different GDM organizations. For example, the United States presidential election requires $1 / 2$ of the vote, while the Chinese Academy of Sciences election requires $2 / 3$ of the vote. In other words, the threshold value of group consensus is affected by the group behaviour or attitude, which has been of research interest in recent consensus reaching process (CRP) models [28]-[32]. Indeed, $\mathrm{Wu}$ et al. in [30] developed an attitudinal trust function to achieve a compromise between the group's aim to reach consensus and the individuals' aim for independence by keeping the inconsistent experts' associated adjustment cost as minimum as possible, which was subsequently translated into an attitudinal consensus degree to determine whether the group needs feedback [31]. Inspired by these approaches, this article investigates an attitudinal consensus threshold (ACT) as a dynamic consensus control mechanism in CRPs, which is able to overcome the shortcoming of traditional methods with regard to their arbitrary selection of the consensus threshold value. Indeed, the proposed ACT is obtained objectively based on the group attitudes, covering the interval from 'minimum 
consensus degree' to 'maximum consensus degree' continuously.

Another key issue in GDM regards the securement of minimum adjustment cost with different consensus thresholds. Recently, Wu et al. in [33] proposed a minimum adjustment cost feedback mechanism based on an optimising model to balance individual adjustment costs and group consensus. Cao et al. in [19] developed a personalized feedback mechanism to reach minimum adjustment cost for individual inconsistent experts. However, the threshold value of group consensus in these studies are fixed beforehand, i.e. it is still static. This article investigates a dynamic minimum adjustment cost optimisation model in which different number of inconsistent experts are determined based on intervals of ACT with the following main result being verified: the adjustment cost monotonically increases with respect to the ACT index. A visual simulation of the consensus degree increment and corresponding adjustment cost with different interval of ACT after the implementation of the feedback process is also presented, which in practice can be used as an aid tool for the group of experts in selecting their most appropriate consensus threshold value for their current GDM scenario to achieve a balance between the individual independence (minimum adjustment cost) and the group aim (achievement of consensus).

The rest of paper is organised as follows: Section II introduces the concept of interval-valued intuitionistic fuzzy set (IVIFS), which is used herein as the representation structure of expert's preferences. Then, the ACT is defined as a consensus control mechanism to conduct the feedback process. In Section III, a dynamic feedback mechanism with minimum adjustment cost based on ACT is proposed. Section IV provides a numerical example comparing the proposed feedback process behaviour with different ACTs. In addition, the relationship between the minimum adjustment cost of both individual and group of experts and the ACT is analysed. Finally, conclusions are pointed out in Section $\mathrm{V}$.

\section{AN ATTITUDINAL CONSENSUS THRESHOLD FOR CONSENSUS CONTROL IN GDM}

As aforementioned, in CRPs there may be conflicts among experts with different backgrounds and knowledge, and therefore it would be necessary to reach group consensus before individual preferences are aggregated. Consensus can be measured at different levels: decision elements (Level 1); decision alternatives (Level 2); and decision matrices (Level 3 ). If all experts have a consensus level 3 greater than or equal to the threshold value of group consensus, then the selection process to achieve a solution to the GDM problem is carried out; otherwise, the experts with consensus level 3 below the threshold value of group consensus, referred to as inconsistent experts, are identified. Then, a feedback mechanism is activated and recommendation advice on how to modify preferences to reach the threshold value of group consensus is provided to the inconsistent experts. Existent consensus models have been designed with fixed given consensus threshold, which results in the associated adjustment cost to be out of control of the corresponding inconsistent experts.
This article, though, proposes a consensus control method by investigating the ACT with minimum adjustment cost to solve the issues on how to obtain the minimum adjustment cost for reaching consensus with different number of feedback experts under different ACT intervals, and how inconsistent experts implement recommendation advice so that they can reach the group consensus threshold value with minimum adjustment cost.

\section{A. Consensus degree with IVIFS}

In GDM problems, it may be difficult for experts to provide accurate preference information due to the uncertainty pervading decision-making problems [34]-[36]. Atanassov and Gargov's [37] interval-valued intuitionistic fuzzy set (IVIFS) reflects the essence of fuzzy information in the objective world intuitively and accurately, with many researchers suggesting using it to describe experts' preference information in fuzzy GDM environments [38]-[40]:

Definition 1 (Interval-Valued Intuitionistic Fuzzy Set (IVIF$\mathrm{S})$ ). Let $X=\left\{x_{1}, x_{2}, \ldots, x_{n}\right\}$ be a finite universe of discourse. An interval-valued intuitionistic fuzzy set (IVIFS) $\widetilde{A}$ over $X$ is given as:

$$
\widetilde{A}=\left\{\left\langle x ; \widetilde{\mu}_{A}(x), \widetilde{\nu}_{A}(x)\right\rangle \mid x \in X\right\}
$$

where $\widetilde{\mu}_{A}(x), \widetilde{\nu}_{A}(x) \in \operatorname{INT}([0,1])$ are respectively the membership interval and the non-membership interval which are subjected to the constraint $0 \leq \sup \widetilde{\mu}_{A}(x)+\sup \widetilde{\nu}_{A}(x) \leq$ $1, \forall x \in X$. An IVIFS can be represented as:

$$
\widetilde{A}=\left\langle x,\left[\widetilde{\mu}_{A}^{L}(x), \widetilde{\mu}_{A}^{U}(x)\right],\left[\widetilde{\nu}_{A}^{L}(x), \widetilde{\nu}_{A}^{U}(x)\right]\right\rangle \mid x \in X
$$

where $0 \leq \widetilde{\mu}_{A}^{U}(x)+\widetilde{\nu}_{A}^{U}(x) \leq 1, \widetilde{\mu}_{A}^{L}(x) \wedge \widetilde{\nu}_{A}^{L}(x) \geq 0$, and $\widetilde{\mu}_{A}^{L}(x), \widetilde{\nu}_{A}^{L}(x)$ and $\widetilde{\mu}_{A}^{U}(x), \widetilde{\nu}_{A}^{U}(x)$ represent the lower and upper limits of $\widetilde{\mu}_{A}(x)$ and $\widetilde{\nu}_{A}(x)$, respectively. The hesitancy degree function of an IVIFS is $\tilde{\pi}_{A}(x)=$ $\left[1-\widetilde{\mu}_{A}^{U}(x)-\widetilde{\nu}_{A}^{U}(x), 1-\widetilde{u}_{A}^{L}(x)-\widetilde{v}_{A}^{L}(x)\right]$."

Based on the Hamming distance, $\mathrm{Xu}$ [39] proposed the following distance between IVIFNs $\alpha_{1}=\left(\left[\mu_{1}^{-}, \mu_{1}^{+}\right],\left[\nu_{1}^{-}, \nu_{1}^{+}\right]\right)$ and $\alpha_{2}=\left(\left[\mu_{2}^{-}, \mu_{2}^{+}\right],\left[\nu_{2}^{-}, \nu_{2}^{+}\right]\right)$:

$$
\begin{aligned}
d\left(\alpha_{1}, \alpha_{2}\right)= & \frac{1}{4}\left(\left|\mu_{1}^{-}-\mu_{2}^{-}\right|+\left|\mu_{1}^{+}-\mu_{2}^{+}\right|+\right. \\
& \left.\left|\nu_{1}^{-}-\nu_{2}^{-}\right|+\left|\nu_{1}^{+}-\nu_{2}^{+}\right|\right)
\end{aligned}
$$

The similarity between IVIFNs can be measured as per the expression [41]:

$$
s\left(\widetilde{\alpha}_{1}, \widetilde{\alpha}_{2}\right)=1-d\left(\widetilde{\alpha}_{1}, \widetilde{\alpha}_{2}\right)
$$

A decision matrix $A=\left(\widetilde{a}_{i j}\right)_{m \times n}$ with IVIFN element $\widetilde{a}_{i j}$ is referred as an interval-valued intuitionistic fuzzy decision matrix (IVIFDM) [42]. Because IFS and IVFS are isomorphic, the operational laws of the latter will be implemented herein [42].

Let $\left\{A^{h}=\left(\widetilde{a}_{i j}^{h}\right)_{m \times n}, h=1, \ldots, k\right\}, \widetilde{a}_{i j}^{h}=\left(\widetilde{\mu}_{i j}^{h}, \widetilde{\nu}_{i j}^{h}\right)$ be the set of IVIFDMs given by a set of experts $E=$ $\left\{E_{1}, E_{2}, \ldots, E_{k}\right\}$, representing their assessments on a set of $m$ alternatives with respect to a set of $n$ criteria. Assuming a 
weight vector of individual experts $w=\left(w_{1}, w_{2}, \ldots, w_{k}\right)^{T}$, the collective aggregated IVIFDM, $\bar{A}=\left(\bar{a}_{i j}\right)_{m \times n}$, has elements defined as follow:

$$
\bar{a}_{i j}=\sum_{h=1}^{k} w_{h} \cdot \widetilde{a}_{i j}^{h}, i=1,2, \ldots, m ; j=1,2, \ldots, n
$$

For each expert, consensus degrees [43], [44] are measured at three levels of an IVIFDM:

1) Consensus degree at the elements level.

$$
C E_{i j}^{h}=s\left(\widetilde{a}_{i j}^{h}, \bar{a}_{i j}\right)=1-d\left(\widetilde{a}_{i j}^{h}, \bar{a}_{i j}\right)
$$

At this level, it is calculated the agreement between an expert and the group on the preference assessment on the alternative $x_{i}$ with respect to the criterion $c_{j}$, which is referred to as the element $\left(x_{i}, c_{j}\right)$.

2) Consensus degree at the alternatives level.

$$
C A_{i}^{h}=\frac{1}{n} \sum_{j=1}^{n} C E_{i j}^{h}
$$

At this level, it is calculated the agreement between an expert and the group on the preference assessment on the alternative $x_{i}$ (with respect to all the criteria).

3) Consensus degree at the decision matrix level.

$$
C D^{h}=\frac{1}{m} \sum_{i=1}^{m} C A_{i}^{h}
$$

At this level, it is calculated the overall agreement between an expert and the group (with respect to all the alternatives and criteria).

\section{B. Threshold value of group consensus with attitude}

In GDM, traditional consensus models usually fix a consensus threshold value $\gamma$ for the whole feedback process, which is subjectively based on the experience or background of the moderator. As aforementioned, the diversity of GDM scenarios requires consensus threshold to be dynamic rather than static. Indeed, in a realistic decision making process, the threshold value will depend on the particular decision-making problem. For example, in the United States, the presidential election requires more than half of the electoral college votes, while the election of an academician of the Chinese Academy of Sciences requires at least two-thirds of the votes. Obviously, how to determine threshold values is a key issue in the field of GDM. Therefore, this article investigates a consensus control method that regards the consensus threshold to be in a continuous interval with lower and upper bounds $\min \left\{C D^{1}, \ldots, C D^{k}\right\}$ and $\max \left\{C D^{1}, \ldots, C D^{k}\right\}$, respectively, and that will be controlled by the experts' attitude. This can be achieved by the implementation of Yager's Ordered Weighted Averaging (OWA) operator [45] with weighting vector derived using O'Hagan's approach [46] with the experts' attitude as the orness value of the OWA operator.

Let $A=\left\{a_{1}, \ldots, a_{n}\right\}$ be a set of real values to aggregate and $\alpha \in[0,1]$ be the orness of the aggregated value, i.e. a value representing the closeness of the aggregated value with respect to the maximum of set $A$. This is formally described in the below definition:

Definition 2 (Attitude-OWA operator (AOWA)). An AOWA operator of dimension $n$ with attitudinal parameter $\alpha$ is an OWA operator of dimension $n$ with weighting vector $W^{\alpha}=\left(w_{1}^{\alpha}, \ldots, w_{n}^{\alpha}\right)$ the solution of the following constrained nonlinear optimisation model:

$$
\begin{aligned}
& \operatorname{Max} \operatorname{disp}(W)=-\sum_{i=1}^{n} w_{i} \cdot \ln w_{i} \\
& \text { s.t. }\left\{\begin{array}{l}
\operatorname{orness}(W)=\sum_{i=1}^{n} \frac{n-i}{n-1} w_{i}=\alpha, 0 \leq \alpha \leq 1 \\
\sum_{i=1}^{n} w_{i}=1, w_{i} \in[0,1], i=(1, \ldots, n)
\end{array}\right.
\end{aligned}
$$

Let $\left\{C D^{1}, \ldots, C D^{k}\right\}$ be a set of experts' consensus degrees. The Attitudinal Consensus Threshold (ACT) is now introduced:

Definition 3 (Attitudinal Consensus Threshold (ACT)). Let $\alpha \in[0,1]$ represent a group of experts' attitudinal value. The group attitudinal consensus threshold (ACT) is associated with the wight $W^{\alpha}$ of $C D$, calculated by expression (9):

$A C T\left(W^{\alpha}\right)=A O W A_{W^{\alpha}}\left(C D^{1}, \ldots, C D^{k}\right)=\sum_{h=1}^{k} w_{h}^{\alpha} \times C D^{\sigma(h)}$

with $C D^{\sigma(h+1)} \leq C D^{\sigma(h)}(\forall h=1, \ldots, k-1)$. Clearly, it is:

1) $A C T\left(W^{0}\right)=\min \left\{C D^{1}, \ldots, C D^{k}\right\}=C D_{\min }$.

2) $A C T\left(W^{1}\right)=\max \left\{C D^{1}, \ldots, C D^{k}\right\}=C D_{\max }$.

The weighting vector $\left(\frac{1}{k}, \frac{1}{k}, \ldots, \frac{1}{k}\right)$ has an orness value of $\frac{1}{2}$ and its corresponding ACT value is $\frac{1}{k} \sum_{h=1}^{k} C D^{k}$. Given a weighting vector $W^{\alpha}$, its corresponding ACT value will be $A C T\left(W^{\alpha}\right)$.

The following properties are verified [47]-[49]:

Proposition 1. $\forall \alpha \in[0,1]: A C T\left(W^{0}\right) \leq A C T\left(W^{\alpha}\right) \leq$ $A C T\left(W^{1}\right)$.

Proposition 2. Let $W^{\alpha}=\left(w_{1}^{\alpha}, w_{2}^{\alpha}, \ldots, w_{k}^{\alpha}\right), W^{\alpha \prime}=$ $\left(w_{1}^{\alpha \prime}, w_{2}^{\alpha \prime}, \ldots, w_{k}^{\alpha \prime}\right)$.

1) If $\frac{w_{h}^{\alpha}}{w_{h+1}^{\alpha}} \geq \frac{w_{h}^{\alpha \prime}}{w_{h+1}^{\alpha}}(\forall h)$, then $A C T\left(W^{\alpha}\right) \geq A C T\left(W^{\alpha^{\prime}}\right)$.

2) If $C D^{h} \neq C D^{l}(\forall h \neq l)$ and $\frac{w_{h}^{\alpha}}{w_{h+1}^{\alpha}} \geq \frac{w_{h}^{\alpha \prime}}{w_{h+1}^{\alpha}}(\forall h)$, then $A C T\left(W^{\alpha}\right)>A C T\left(W^{\alpha \prime}\right)$.

Corollary 1. Given a weighting vector $W^{\alpha}=$ $\left(w_{1}^{\alpha}, w_{2}^{\alpha}, \ldots, w_{k}^{\alpha}\right)$ :

1) If $w_{1}^{\alpha} \geq w_{2}^{\alpha} \geq \ldots \geq w_{k}^{\alpha}$, then $\operatorname{ACT}\left(W^{\alpha}\right) \geq$ $\frac{1}{k} \sum_{h=1}^{k} C D^{k}$

2) If $w_{1}^{\alpha} \leq w_{2}^{\alpha} \leq \ldots \leq w_{k}^{\alpha}$, then $\operatorname{ACT}\left(W^{\alpha}\right) \leq$ $\frac{1}{k} \sum_{h=1}^{k} C D^{k}$

In GDM, the group ACT acts as a parameter to control when the feedback process needs to be activated. On the one hand, when all experts' $\mathrm{CD}$ values above the ACT value, the feedback process is not needed and there is sufficient 
consensus among the group of experts to achieve a solution of consensus. When this is not the case, i.e. when some experts have a $\mathrm{CD}$ value below the ACT, who are referred to as inconsistent experts, the feedback process is activated and recommendations are provided to the inconsistent experts to help them increase their $\mathrm{CD}$ values so that the ACT is reached. When $\alpha=0$, the ACT value will be $C D_{\min }$ and therefore all experts $\mathrm{CD}$ values will be greater than or equal to the ACT; thus, in this case, the feedback process is not activated. On the other hand, when $\alpha=1$, the ACT will be $C D_{\max }$ and, unless all experts have the same $C D$ value, the feedback process is activated. Obviously, the number of inconsistent experts will be dependent on the value of the ACT. Summarising the above, denoting the ACT value as $\gamma=A C T\left(W^{\alpha}\right), \alpha \in[0,1]$, then when $C D_{h} \geq \gamma(\forall h)$, the resolution process of the GDM is carried out. Otherwise, the feedback process is required. It is noteworthy that the ACT proposed in this article aims to observe the changes in the number of experts and costs in the feedback process by changing the value of $\alpha$, so that individuals and groups can coordinate depending on their own willing regarding group consistency and individual independence, as the numerical analysis will show.

\section{DYNAMIC FEEDBACK MECHANISM BASED ON ATTITUDINAL CONSENSUS THRESHOLD}

This section introduces a dynamic minimum adjustment cost feedback mechanism to provide personalised advice for inconsistent experts based on an optimisation model to determine the boundary feedback parameter. Its novelty is that the implementation of threshold value $A C T \in\left[C D_{\min }, C D_{\max }\right]$ is used to control the feedback process dynamically and to allow the inconsistent experts to know how to adopt the personalised advice if they are willing to reach the ACT value. The attitudinal consensus threshold based dynamic minimum adjustment cost feedback mechanism for reaching consensus is illustrated in Fig.1.

\section{A. Dynamic identification mechanism of inconsistent decision matrix elements}

Algorithm 1 identifies the inconsistent experts, and their alternatives and corresponding elements with $\mathrm{CE}$ and $\mathrm{CA}$ values below the ACT value.

\section{B. Advice generation with boundary feedback parameter}

For the identified elements in $A P S$, the corresponding inconsistent expert receives the following advice: "Evaluation value $a_{i j}^{\sigma(h)}=\left(\widetilde{\mu}_{i j}^{\sigma(h)}, \widetilde{\nu}_{i j}^{\sigma(h)}\right)$ should be closer to $\vartheta_{i j}^{\sigma(h)}=$ $\left(\vartheta \widetilde{\mu}_{i j}^{\sigma(h)}, \vartheta \widetilde{\nu}_{i j}^{\sigma(h)}\right)$,

$$
\begin{aligned}
\left(\vartheta \mu_{i j}^{\sigma(h)}, \vartheta \nu_{i j}^{\sigma(h)}\right)= & \left(\left(1-\delta_{\sigma(h)}\right) \cdot \mu_{i j}^{\sigma(h)}+\delta_{\sigma(h)} \cdot \overline{\mu_{i j}},\right. \\
& \left.\left(1-\delta_{\sigma(h)}\right) \cdot \nu_{i j}^{\sigma(h)}+\delta_{\sigma(h)} \cdot \overline{\nu_{i j}}\right)
\end{aligned}
$$

where $\delta_{\sigma(h)} \in[0,1]$ is a feedback parameter used to control the extent of the change from the original evaluation $a_{i j}^{\sigma(h)}$ $\overline{\text { Algorithm } 1 \text { Dynamic identification mechanism of consensus }}$ degree at three level

\section{Input:}

The experts' consensus degree at the three levels: $C D^{\sigma(h)}, C A_{i}^{\sigma(h)}, C E_{i j}^{\sigma(h)} \quad$ with $C D^{\sigma(h+1)} \leq$ $C D^{\sigma(h)}(\forall h=1, \ldots, k-1)$;

The attitudinal parameter: $\alpha \in[0,1]$; The attitudinal consensus threshold: $A C T\left(W^{\alpha}\right)$;

\section{Output:}

Inconsistent experts, alternatives and elements: $E X P C H$, $A L T, A P S$;

$$
\begin{gathered}
E X P C H=\left\{\sigma(h) \mid C D^{\sigma(h)}<A C T\left(W^{\alpha}\right)\right\} \\
A L T=\left\{(\sigma(h), i) \mid \sigma(h) \in E X P C H \wedge C A_{i}^{\sigma(h)}<A C T\left(W^{\alpha}\right)\right\}
\end{gathered}
$$

$$
A P S=\left\{(\sigma(h), i, j) \mid(\sigma(h), i) \in A L T \wedge C E_{i j}^{\sigma(h)}<A C T\left(W^{\alpha}\right)\right\}
$$

to the collective evaluation $\bar{a}_{i j}$. When the feedback parameter value is $\delta_{\sigma(h)}=1$, the original assessment of the inconsistent experts is completely replaced by the collective assessment, while when $\delta_{\sigma(h)}=0$ the experts original preference is unchanged. The differences between the original assessments and the advised assessments can be regarded as the adjustment cost faced by the inconsistent experts. The adjustment cost increases with the feedback parameter. The traditional feedback process usually chooses the same fixed feedback parameter value for all inconsistent experts, which could lead to a total adjustment cost (TC) for some inconsistent experts higher than the actual demand.

$$
\begin{aligned}
T C & =\sum_{(\sigma(h), i, j) \in A P S}\left|a_{i j}^{\sigma(h)}-\vartheta_{i j}^{\sigma(h)}\right| \\
& =\sum_{\sigma(h), i, j \in A P S} \delta_{\sigma(h)}\left|a_{i j}^{\sigma(h)}-\overline{a_{i j}}\right|
\end{aligned}
$$

Thus, it is key for the interaction in GDM to find appropriate feedback parameters for the inconsistent experts to minimise their adjustment costs.

Ben-Arieh and Easton [50] provided a comprehensive analysis of the cost of reaching consensus. Other minimum adjustment cost optimal models for CRP have been proposed [33], [51]-[55]. However, these models, as aforementioned, rely on a static feedback parameter, and therefore cannot properly reflect the consistency requirements of the actual GDM problem. To overcome this issue, the following dynamic 
2

3

4

5

6

7

8

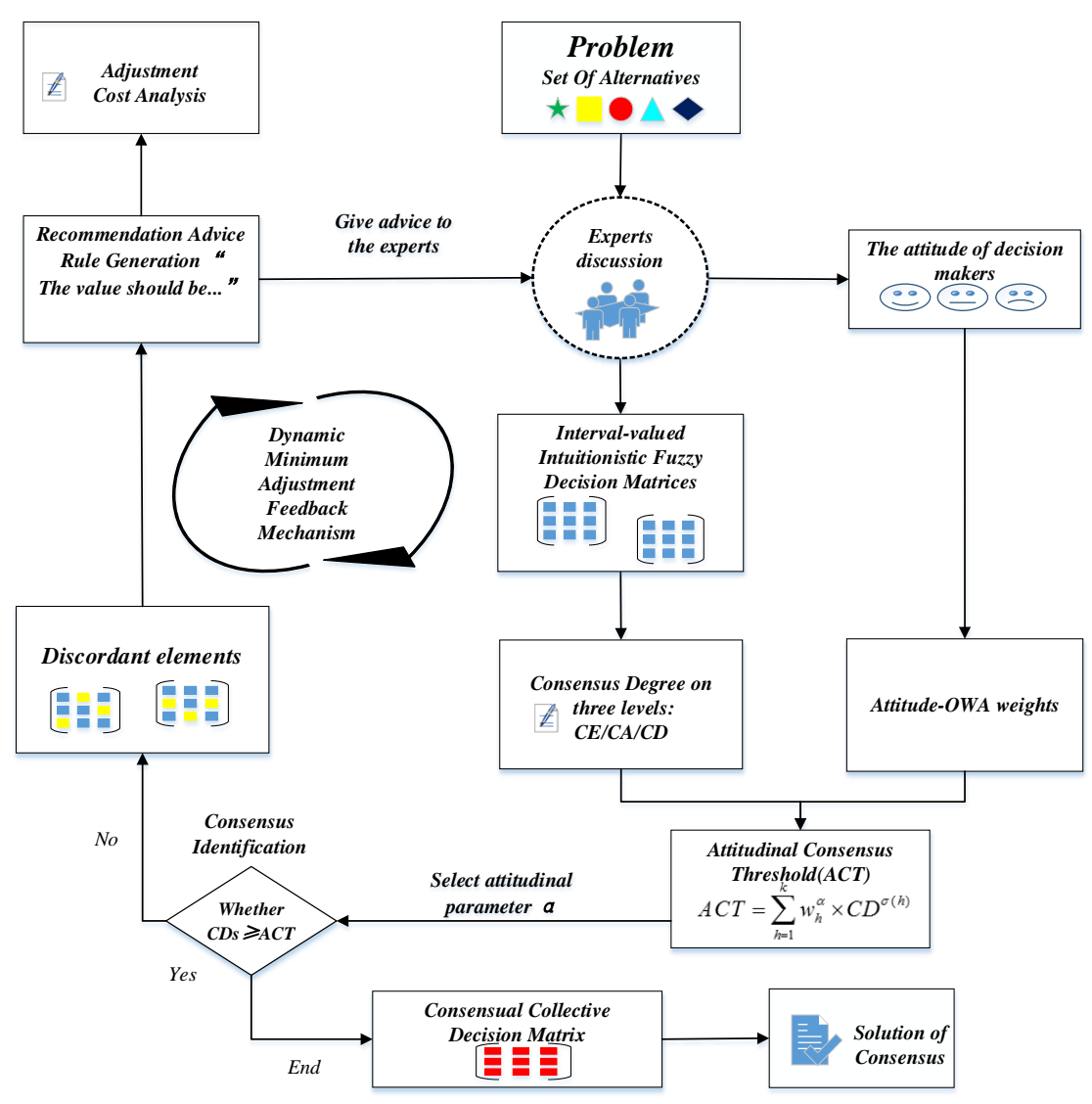

Fig. 1: ACT based dynamic feedback mechanism for consensus

optimisation total cost model is proposed:

$$
\begin{aligned}
& \operatorname{Min} T C=\sum_{(\sigma(h), i, j) \in A P S}\left|a_{i j}^{\sigma(h)}-\vartheta_{i j}^{\sigma(h)}\right| \\
& \text { s.t. }\left\{\begin{array}{l}
\vartheta_{i j}^{\sigma(h)}=\left(1-\delta_{\sigma(h)}\right) \cdot a_{i j}^{\sigma(h)}+\delta_{\sigma(h)} \cdot \overline{a_{i j}} \\
A C T\left(W^{\alpha}\right) \in\left[C D_{\min }, C D_{\max }\right] \\
C D^{\sigma(h)}<A C T\left(W^{\alpha}\right)(\sigma(h) \in A P S) \\
C D^{\sigma(h)}=A C T\left(W^{\alpha}\right) \\
\delta_{\sigma(h)} \leq \delta_{\sigma(h+1)} \\
0 \leq \delta_{\sigma(h)} \leq 1
\end{array}\right.
\end{aligned}
$$

where $C D^{\sigma(h)}$ represents the $\mathrm{CD}$ value before feedback. $\overline{C D^{\sigma(h)}}$ represents the new $\mathrm{CD}$ value after one round of feedback process. According to expression (9)-(10) and expression (15), the above optimisation model can be rewritten as follows:

$$
\begin{aligned}
& \operatorname{Min} T C=\sum_{\sigma(h), i, j \in A P S} \delta_{\sigma(h)}\left|a_{i j}^{\sigma(h)}-\overline{a_{i j}}\right| \\
& \text { s.t. }\left\{\begin{array}{l}
\vartheta_{i j}^{\sigma(h)}=\left(1-\delta_{\sigma(h)}\right) \cdot a_{i j}^{\sigma(h)}+\delta_{\sigma(h)} \cdot \overline{a_{i j}} \\
A C T\left(W^{\alpha}\right)=\sum_{h=1}^{k} w_{h}^{\alpha} \times C D^{\sigma(h)} \\
\left\{\begin{array}{l}
\operatorname{Max} \operatorname{disp}(W)=-\sum_{h=1}^{k} w_{h} \cdot \ln w_{h} \\
\text { s.t. }\left\{\begin{array}{l}
k=1 \\
\sum_{h=1}^{k} w_{h}=1 \\
k=1
\end{array} w_{h}, 0 \leq \alpha \leq 1\right. \\
\\
\frac{C D^{\sigma(h)}<A C T\left(W^{\alpha}\right)(\sigma(h) \in A P S)}{C D^{\sigma(h)}=A C T\left(W^{\alpha}\right)} \\
\delta_{\sigma(h)} \leq \delta_{\sigma(h+1)} \\
0 \leq \delta_{\sigma(h)} \leq 1
\end{array}\right.
\end{array}\right.
\end{aligned}
$$

Notably, there will be the case that the group requires higher consensus degree than the consensus threshold of $A C T\left(W^{1}\right)$. In this case, all experts are identified as inconsistent experts 
and the optimized feedback model is re-established in the following expression (18):

$$
\begin{aligned}
& \operatorname{Min} T C=\sum_{\sigma(h), i, j \in A P S} \delta_{\sigma(h)}\left|a_{i j}^{\sigma(h)}-\overline{a_{i j}}\right| \\
& \text { s.t. }\left\{\begin{array}{l}
\vartheta_{i j}^{\sigma(h)}=\left(1-\delta_{\sigma(h)}\right) \cdot a_{i j}^{\sigma(h)}+\delta_{\sigma(h)} \cdot \overline{a_{i j}} \\
C D^{\sigma(h)}<\bar{\gamma}(\sigma(h) \in A P S) \\
\overline{C D^{\sigma(h)}}=\bar{\gamma} \\
\delta_{\sigma(h)} \leq \delta_{\sigma(h+1)} \\
0 \leq \delta_{\sigma(h)} \leq 1
\end{array}\right.
\end{aligned}
$$

where $\bar{\gamma} \in\left(A C T\left(W^{1}\right), 1\right]$.

\section{Algorithm for dynamic feedback mechanism}

The proposed consensus model is provided below in Algorithm 2:

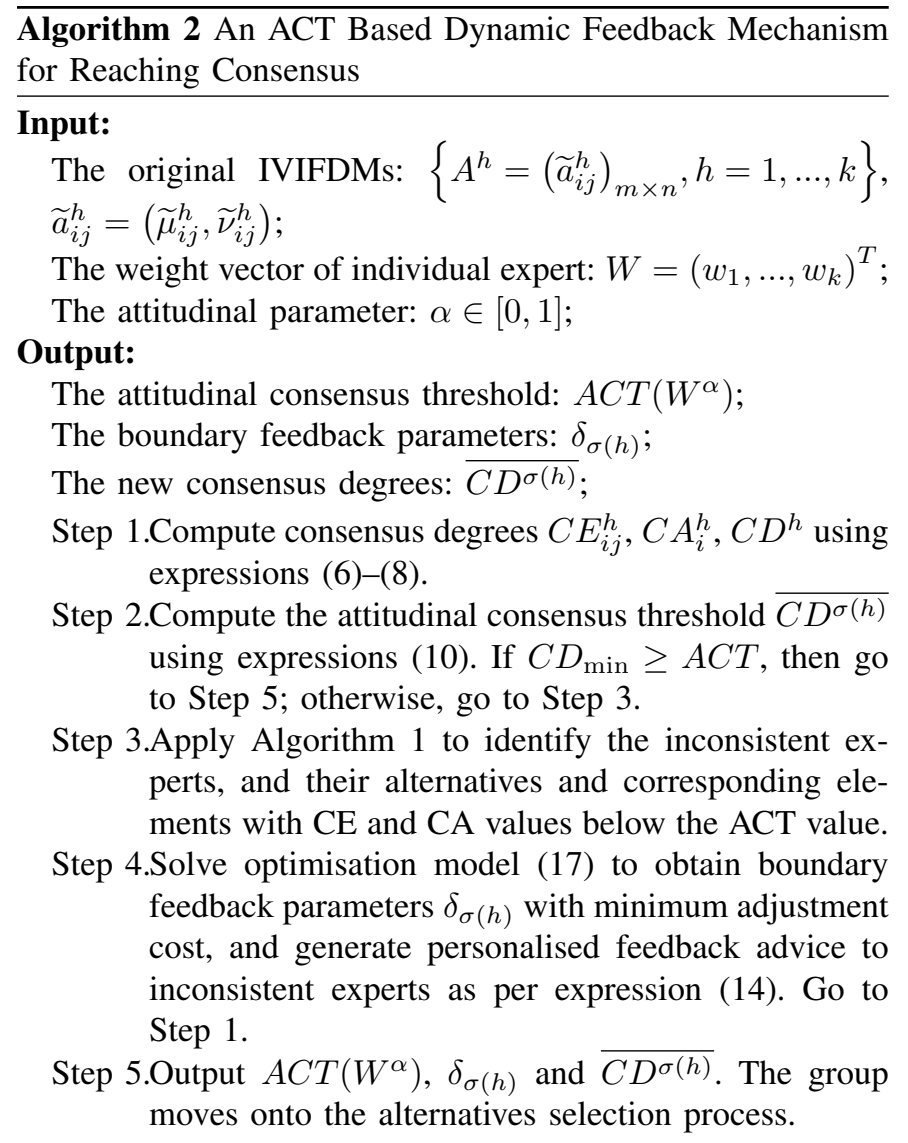

It is worth noting that the group consensus reaching cannot guarantee that the decision result must be correct. Indeed, if the preference information provided by most experts is wrong, the final decision-making result cannot be guaranteed to be correct. The visual feedback simulation presented in this article aims to provide some personalized advice to experts who are inconsistent with the majority so that the decision can be carried out smoothly. Therefore, this article mainly focuses on the achievement of group consensus. Although this is the ultimate goal of any decision-making, it cannot guarantee the final quality of the decision. However, in certain specific decision-making environments, reaching consensus is necessary. For example, in a partnership, each partner is responsible for the profits and losses of their company. In this case, an agreement must be reached before executing decision process. Similarly, when formulating a surgical plan, each expert must agree on the operation before it can be performed.

\section{NUMERICAL ANALYSIS}

\section{A. The construction of dynamic decision-making}

The emergency department of a manufacturing enterprise wants to make appropriate emergency scheme to deal with emergencies. Due to the timeliness of emergency decisionmaking (EDM) problem, the consistency requirements may be different. For example, if the incident is urgent, a few experts agree that the scheme will be conducted. While if the incident has a buffer time, maybe most experts agree that the scheme will be implemented. Therefore, the consistency requirements are changing constantly with different EDM problems. After pre evaluation, four plans $\left\{M_{1}, M_{2}, M_{3}, M_{4}\right\}$ have remained as alternatives for further evaluation. Three criteria $\left\{N_{1}, N_{2}, N_{3}\right\}$ are considered as follows: $N_{1}$, emergency response; $N_{2}$, safeguard measures; $N_{3}$, material and equipment support. Four experts $\left\{e_{1}, e_{2}, e_{3}, e_{4}\right\}$ from emergency field organize this evaluation (it is assumed that each expert has rich experience, and has intention to reach consensus on the choice of alternatives), providing the following IVIFDMs:

$$
\begin{aligned}
& A^{1}=\left(\begin{array}{ccc}
N_{1} & N_{2} & N_{3} \\
M_{1}\langle[0.4,0.5],[0.1,0.3]\rangle & \langle[0.1,0.3],[0.1,0.4]\rangle & \langle[0.4,0.5],[0.2,0.4]\rangle \\
M_{2}\langle[0.1,0.4],[0.5,0.6]\rangle & \langle[0.1,0.4],[0.2,0.5]\rangle & \langle[0.3,0.6],[0.2,0.3]\rangle \\
M_{3}\langle[0.1,0.4],[0.4,0.5]\rangle & \langle[0.3,0.6],[0.1,0.2]\rangle & \langle[0.4,0.6],[0.1,0.2]\rangle \\
M_{4}\langle[0.3,0.7],[0.2,0.3]\rangle & \langle[0.2,0.3],[0.2,0.3]\rangle & \langle[0.1,0.5],[0.3,0.4]\rangle
\end{array}\right) \\
& A^{2}=\left(\begin{array}{ccc}
N_{1} & N_{2} & N_{3} \\
M_{1}\langle[0.2,0.3],[0.5,0.6]\rangle & \langle[0.3,0.4],[0.5,0.6]\rangle & \langle[0.1,0.2],[0.6,0.7]\rangle \\
M_{2}\langle[0.3,0.4],[0.1,0.2]\rangle & \langle[0.5,0.6],[0.2,0.3]\rangle\langle[0.6,0.7],[0.1,0.2]\rangle \\
M_{3}\langle[0.2,0.4],[0.5,0.6]\rangle\langle[0.1,0.2],[0.4,0.6]\rangle & \langle[0.2,0.4],[0.4,0.6]\rangle \\
M_{4}\langle[0.2,0.4],[0.4,0.5]\rangle\langle[0.4,0.5],[0.1,0.2]\rangle & \langle[0.1,0.2],[0.6,0.7]\rangle
\end{array}\right)
\end{aligned}
$$

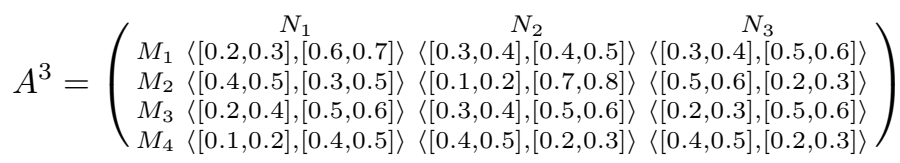

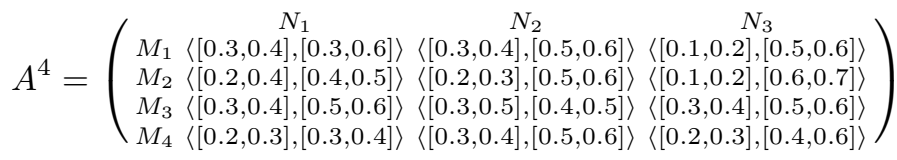

\section{B. The computation of ACT}

(1) This article assumes equal importance degree for each expert: $w=\left(\frac{1}{4}, \frac{1}{4}, \frac{1}{4}, \frac{1}{4}\right)^{T}$. Therefore, all the decision matrices $A^{h}$ of the four experts can be aggregated into a new decision matrix $\bar{A}$ :

(2) Consensus degrees computation.

Level 1. The consensus degree of decision elements:

$$
C E^{1}=\left(\begin{array}{lll}
0.8063 & 0.8438 & 0.8063 \\
0.8750 & 0.9000 & 0.9250 \\
0.9375 & 0.8125 & 0.7813 \\
0.8375 & 0.9125 & 0.9000
\end{array}\right)
$$




$$
\begin{gathered}
\bar{A}=\left(\begin{array}{l}
M_{1}\langle[0.275,0.375],[0.375,0.550]\rangle \\
M_{2}\langle[0.250,0.425],[0.325,0.450]\rangle \\
M_{3}\langle[0.200,0.400],[0.475,0.575]\rangle \\
M_{4}\langle[0.200,0.400],[0.325,0.425]\rangle
\end{array}\right. \\
C E^{2}=\left(\begin{array}{lll}
0.9188 & 0.9313 & 0.8688 \\
0.8625 & 0.7625 & 0.8125 \\
0.9875 & 0.8625 & 0.9438 \\
0.9625 & 0.8875 & 0.8250
\end{array}\right) \\
C E^{3}=\left(\begin{array}{lll}
0.8688 & 0.9688 & 0.9438 \\
0.9250 & 0.7875 & 0.9125 \\
0.9875 & 0.9125 & 0.8938 \\
0.8875 & 0.9375 & 0.8250
\end{array}\right) \\
C E^{4}=\left(\begin{array}{lll}
0.9563 & 0.9313 & 0.9188 \\
0.9500 & 0.9375 & 0.6875 \\
0.9625 & 0.9500 & 0.9313 \\
0.9625 & 0.8625 & 0.9500
\end{array}\right)
\end{gathered}
$$

Level 2. The consensus degree of decision alternatives:

$$
\begin{array}{llll}
C A^{1}=\left(\begin{array}{llll}
0.8188, & 0.9000, & 0.8438, & 0.8833
\end{array}\right) ; \\
C A^{2}=\left(\begin{array}{llll}
0.9063, & 0.8125, & 0.9313, & 0.8917
\end{array}\right) ; \\
C A^{3}=\left(\begin{array}{llll}
0.9271, & 0.8750, & 0.9313, & 0.8833
\end{array}\right) ; \\
C A^{4}=\left(\begin{array}{llll}
0.9354, & 0.8583, & 0.9479, & 0.9250
\end{array}\right)
\end{array}
$$

Level 3. The consensus degree of decision matrix:

$$
\begin{aligned}
& C D^{1}=0.861, C D^{2}=0.885 \\
& C D^{3}=0.904, C D^{4}=0.917
\end{aligned}
$$

From model (10), we get:

$$
\begin{aligned}
& C D^{\sigma(1)}=C D^{4}, C D^{\sigma(2)}=C D^{3} ; \\
& C D^{\sigma(3)}=C D^{2}, C D^{\sigma(4)}=C D^{1}
\end{aligned}
$$

(3) The weights $w_{h}(h=1,2,3,4)$ are computed by model (9) with different attitudinal parameter $\alpha$ and the ACT representing the group consensus threshold values from model (10) are given in Table I, which clearly reflect the dynamic changes of ACT with respect to the attitudinal parameter $\alpha$ from $C D_{\min }$ to $C D_{\max }$.

\section{Analysis of feedback process with different ACT}

(1) Taking $\alpha=0.2$, we get $A C T\left(W^{0.2}\right)=0.875$ from Table I.

The sets of 3-tuple identified as contributing less to consensus are:

$$
\begin{aligned}
A P S= & (1,1,1),(1,1,2),(1,1,3),(1,2,1), \\
& (1,3,2),(1,3,3),(1,4,1)\}
\end{aligned}
$$

(2) With the threshold value $A C T\left(W^{0.2}\right)$, expert $e_{1}$ participates in the feedback process at this point. From the model (17), we get the following model (19).

$$
\begin{aligned}
& \operatorname{Min} T C=\sum_{\sigma(h), i, j \in A P S} \delta_{\sigma(4)} \cdot\left|a_{i j}^{\sigma(4)}-\overline{a_{i j}}\right|
\end{aligned}
$$

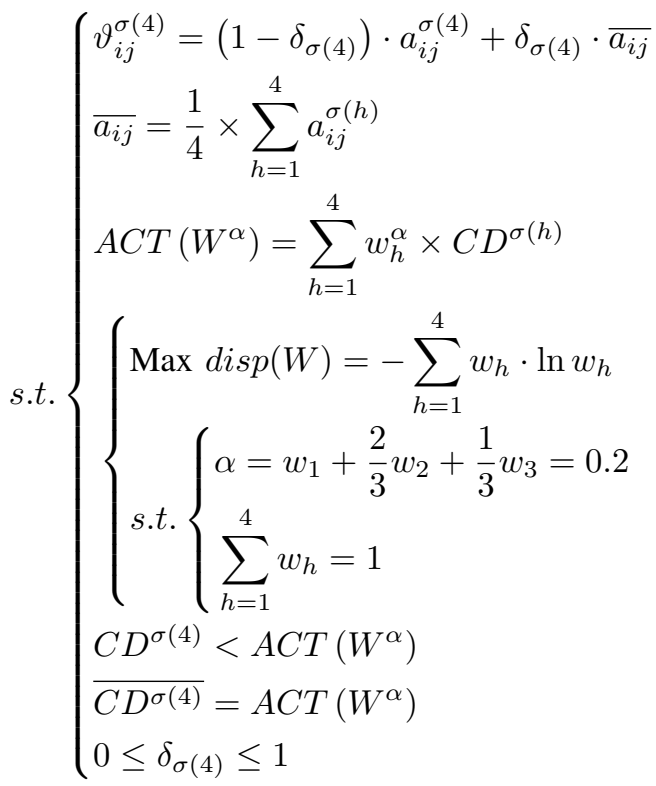

By solving the model (19), we get the boundary feedback parameter $\delta_{1_{\text {min }}}=\delta_{\sigma(4)}=0.175$.

(3) Taking the feedback parameter value of $\delta_{1_{\min }}=0.175$, the feedback mechanism would provide the following recommendations to expert $e_{1}$ :

- Your preference value of $\vartheta_{11}^{1}$ should be closer to $\left\langle\left[\begin{array}{lll}0.378 & 0.478\end{array}\right],\left[\begin{array}{lll}0.148 & 0.344\end{array}\right]\right\rangle$;

- Your preference value of $\vartheta_{12}^{1}$ should be closer to $\left\langle\left[\begin{array}{lll}0.126 & 0.313\end{array}\right],\left[\begin{array}{lll}0.148 & 0.422\end{array}\right]\right\rangle$;

- Your preference value of $\vartheta_{13}^{1}$ should be closer to $\left\langle\left[\begin{array}{ll}0.369 & 0.469\end{array}\right],\left[\begin{array}{lll}0.244 & 0.431\end{array}\right]\right\rangle$;

- Your preference value of $\vartheta_{21}^{1}$ should be closer to $\left\langle\left[\begin{array}{ll}0.126 & 0.404\end{array}\right],\left[\begin{array}{lll}0.469 & 0.574\end{array}\right]\right\rangle$;

- Your preference value of $\vartheta_{32}^{1}$ should be closer to $\left\langle\left[\begin{array}{ll}0.291 & 0.569\end{array}\right],\left[\begin{array}{lll}0.144 & 0.248\end{array}\right]\right.$;

- Your preference value of $\vartheta_{33}^{1}$ should be closer to $\left\langle\left[\begin{array}{lll}0.378 & 0.569\end{array}\right],\left[\begin{array}{lll}0.148 & 0.253\end{array}\right]\right\rangle$;

- Your preference value of $\vartheta_{41}^{1}$ should be closer to $\left\langle\left[\begin{array}{lll}0.282 & 0.647\end{array}\right],\left[\begin{array}{lll}0.222 & 0.322\end{array}\right]\right\rangle$.

(4) After expert $e_{1}$ revisits his/her evaluations and implements the recommended IVIFNs, the new decision matrix would be: $A_{\alpha=0.2}^{1}$.

The new $C D s$ are computed:

$$
\begin{aligned}
& \overline{C D^{\sigma(4)}}=0.875 ; \overline{C D^{\sigma(3)}}=0.889 ; \\
& \overline{C D^{\sigma(2)}}=0.907 ; \overline{C D^{\sigma(1)}}=0.918
\end{aligned}
$$

(5) A visual feedback process simulation is shown in Fig.2 (a-b), which generates a graphical simulation of $C D s$ conditions. It can be seen that the $C D s$ values of all 
TABLE I: Attitudinal Consensus Threshold with different $\alpha$

\begin{tabular}{cccccccccccc}
\hline$\alpha$ & 0 & 0.1 & 0.2 & 0.3 & 0.4 & 0.5 & 0.6 & 0.7 & 0.8 & 0.9 & 1 \\
\hline$w_{1}$ & 0 & 0.010 & 0.045 & 0.098 & 0.167 & 0.250 & 0.347 & 0.461 & 0.597 & 0.764 & 1 \\
$w_{2}$ & 0 & 0.043 & 0.107 & 0.165 & 0.213 & 0.250 & 0.272 & 0.276 & 0.252 & 0.182 & 0 \\
$w_{3}$ & 0 & 0.182 & 0.252 & 0.276 & 0.272 & 0.250 & 0.213 & 0.165 & 0.107 & 0.043 & 0 \\
$w_{4}$ & 1 & 0.764 & 0.597 & 0.461 & 0.347 & 0.250 & 0.167 & 0.098 & 0.045 & 0.010 & 0 \\
$A C T$ & 0.861 & 0.868 & 0.875 & 0.881 & 0.886 & 0.892 & 0.896 & 0.903 & 0.908 & 0.912 & 0.917 \\
\hline
\end{tabular}

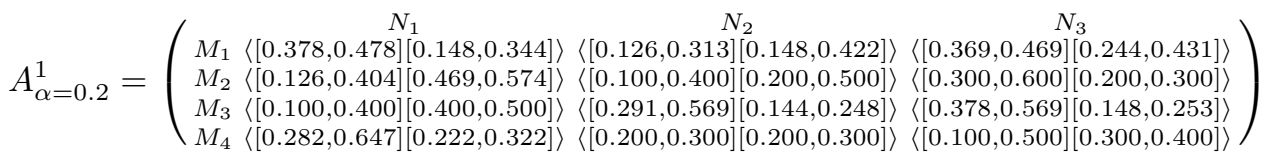

experts before and after feedback when the threshold value $A C T\left(W^{0.2}\right)=0.875$. Fig.2(a) depicts the $C D s$ values of experts before feedback, while Fig.2(b) depicts the $C D s$ values of experts (coloured) after the feedback process, with the value of $C D_{1}$ increasing from 0.861 to 0.875 , which is just equal to $A C T\left(W^{0.2}\right)$.

(6) Supposing $\alpha=0.6$, we get $A C T\left(W^{0.6}\right)=0.896$ from Table I, and experts $e_{1}$ and $e_{2}$ are identified as inconsistent experts. We get the following model (20).

$$
\begin{aligned}
& \operatorname{Min} T C=\sum_{\sigma(h), i, j \in A P S} \delta_{\sigma(4)} \cdot\left|a_{i j}^{\sigma(4)}-\overline{a_{i j}}\right|+ \\
& \delta_{\sigma(3)} \cdot\left|a_{i j}^{\sigma(3)}-\overline{a_{i j}}\right|
\end{aligned}
$$

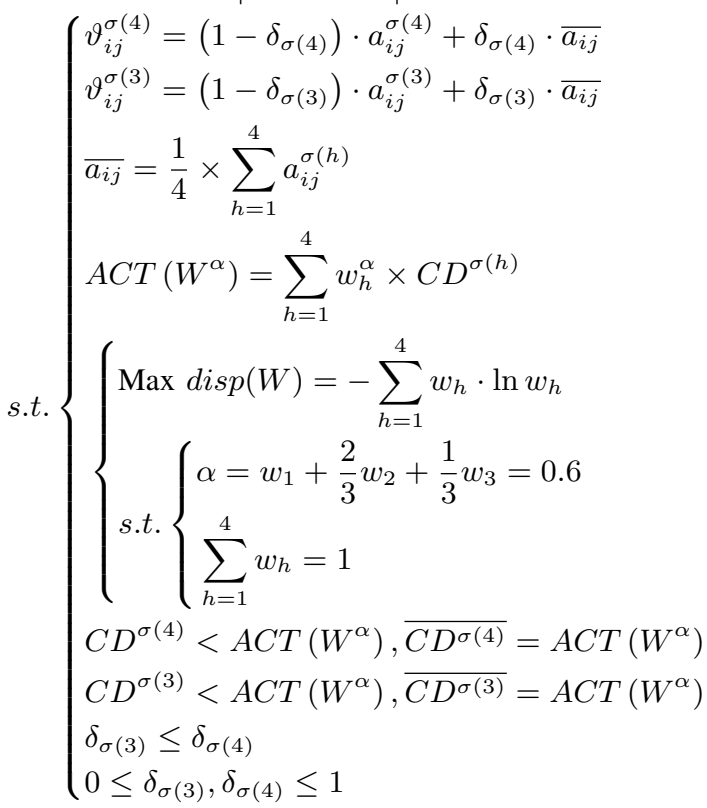

By solving the model (20), we get the boundary feedback parameter $\delta_{1_{\min }}=\delta_{\sigma(4)}=0.445$ and $\delta_{2_{\min }}=\delta_{\sigma(3)}=$ 0.019 .

By identifying inconsistent decision matrix elements and generating advice with boundary feedback parameters, the new decision matrix would be: $A_{\alpha=0.6}^{1}, A_{\alpha=0.6}^{2}$. The new CDs are computed:

$$
\begin{aligned}
& \overline{C D^{\sigma(4)}}=0.896 ; \overline{C D^{\sigma(3)}}=0.896 ; \\
& \overline{C D^{\sigma(2)}}=0.912 ; \overline{C D^{\sigma(1)}}=0.92
\end{aligned}
$$

Fig.3 depicts the $C D s$ values of all experts before and after feedback when the threshold value $A C T\left(W^{0.6}\right)=$ 0.896. Fig.3(a) depicts the $C D$ on the preference of experts before feedback, while Fig.3(b) depicts the $C D$ values of experts (coloured) after the feedback process, with the value of $C D_{1}$ and $C D_{2}$ increasing from 0.861 and 0.855 , respectively, to 0.896 , which is equal to $A C T\left(W^{0.6}\right)$.

(7) Assuming $\alpha=1$ with $A C T\left(W^{1}\right)=0.917$ from Table I, experts $e_{1}, e_{2}$ and $e_{3}$ are identified as inconsistent experts. We get the following model (21).

$$
\begin{aligned}
& \text { Min } T C=\sum_{h, i, j \in A P S} \delta_{\sigma(4)} \cdot\left|a_{i j}^{\sigma(4)}-\overline{a_{i j}}\right|+ \\
& \delta_{\sigma(3)} \cdot\left|a_{i j}^{\sigma(3)}-\overline{a_{i j}}\right|+\delta_{\sigma(2)} \cdot\left|a_{i j}^{\sigma(2)}-\overline{a_{i j}}\right| \\
& \text { s.t. }\left\{\begin{array}{l}
\vartheta_{i j}^{\sigma(4)}=\left(1-\delta_{\sigma(4)}\right) \cdot a_{i j}^{\sigma(4)}+\delta_{\sigma(4)} \cdot \overline{a_{i j}} \\
\vartheta_{i j}^{\sigma(3)}=\left(1-\delta_{\sigma(3)}\right) \cdot a_{i j}^{\sigma(3)}+\delta_{\sigma(3)} \cdot \overline{a_{i j}} \\
\vartheta_{i j}^{\sigma(2)}=\left(1-\delta_{\sigma(2)}\right) \cdot a_{i j}^{\sigma(2)}+\delta_{\sigma(2)} \cdot \overline{a_{i j}} \\
\overline{a_{i j}}=\frac{1}{4} \times \sum_{h=1}^{4} a_{i j}^{\sigma(h)} \\
A C T\left(W^{\alpha}\right)=\sum_{h=1}^{4} w_{h}^{\alpha} \times C D^{\sigma(h)} \\
\operatorname{Max} d i s p(W)=-\sum_{h=1}^{4} w_{h} \cdot \ln w_{h} \\
\quad\left(\alpha=w_{1}+\frac{2}{3} w_{2}+\frac{1}{3} w_{3}=1\right. \\
s . t .\left\{\begin{array}{l}
4 \\
\sum_{h=1} w_{h}=1
\end{array}\right. \\
C D^{\sigma(4)}<A C T\left(W^{\alpha}\right), \overline{C D^{\sigma(4)}}=A C T\left(W^{\alpha}\right) \\
C D^{\sigma(3)}<A C T\left(W^{\alpha}\right), \overline{C D^{\sigma(3)}}=A C T\left(W^{\alpha}\right) \\
D^{\sigma(2)}<A C T\left(W^{\alpha}\right), \overline{C D^{\sigma(2)}}=A C T\left(W^{\alpha}\right) \\
0 \leq \delta_{\sigma(3)}, \delta_{\sigma(3)} \leq \delta_{\sigma(4)}
\end{array}\right.
\end{aligned}
$$

By solving the model (21), we get the boundary feedback parameter $\delta_{1_{\min }}=\delta_{\sigma(4)}=0.552, \delta_{2_{\min }}=\delta_{\sigma(3)}=0.284$ and $\delta_{3_{\min }}=\delta_{\sigma(2)}=0.047$.

By identifying inconsistent decision matrix elements and generating advice with boundary feedback parameters, the 


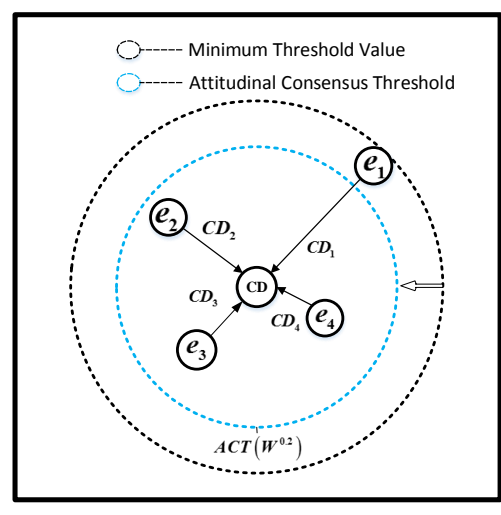

(a) Expert CDs

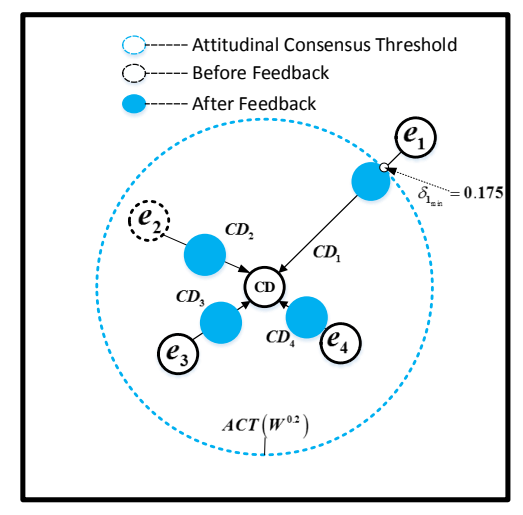

(b) CDs after feedback

Fig. 2: Consensus simulation of expert $e_{1}$ by dynamic feedback mechanism.

$$
\begin{aligned}
& A_{\alpha=0.6}^{1}=\left(\begin{array}{ccc}
N_{1} & N_{2} & N_{3} \\
M_{2}\langle[0.344,0.444][0.222,0.411]\rangle & \langle[0.167,0.333][0.222,0.456]\rangle & \langle[0.322,0.422][0.311,0.478]\rangle \\
M_{3}\langle[0.100,0.411][0.422,0.533]\rangle & \langle[0.100,0.400][0.200,0.500]\rangle & \langle[0.300,0.600][0.200,0.300]\rangle \\
M_{4}\langle[0.255,0.565][0.256,0.300]\rangle & \langle[0.278,0.522][0.211,0.322]\rangle & \langle[0.344,0.522][0.222,0.334]\rangle
\end{array}\right)
\end{aligned}
$$

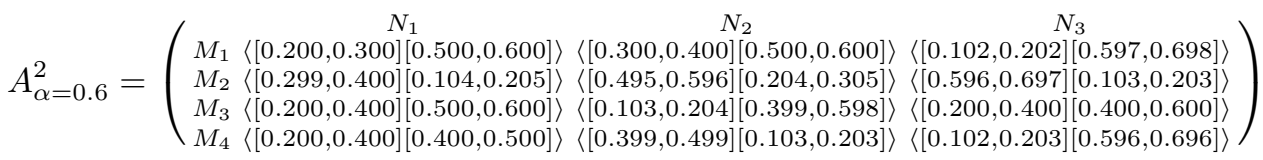

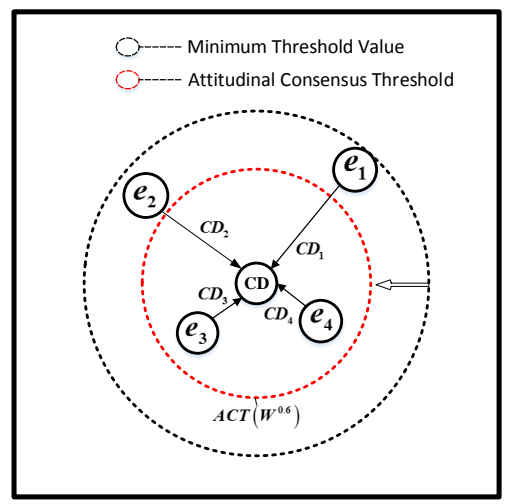

(a) Expert CDs

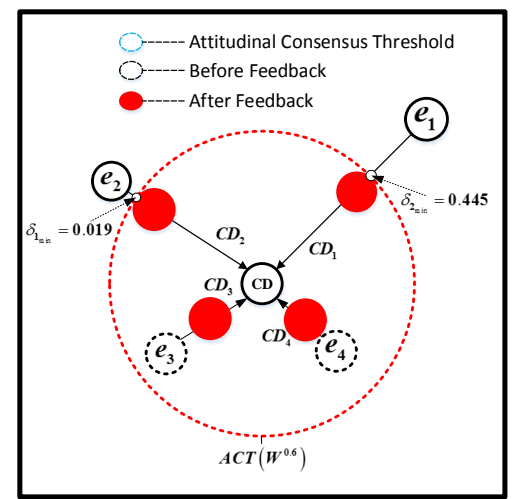

(b) CDs after feedback

Fig. 3: Consensus simulation of experts $e_{1}$ and $e_{2}$ by dynamic feedback mechanism.

new decision matrix would be: $A_{\alpha=1}^{1}, A_{\alpha=1}^{2}$ and $A_{\alpha=1}^{3}$. The new $C D s$ are computed:

$$
\begin{aligned}
& \overline{C D^{\sigma(4)}}=0.917 ; \overline{C D^{\sigma(3)}}=0.917 ; \\
& \overline{C D^{\sigma(2)}}=0.917 ; \overline{C D^{\sigma(1)}}=0.924
\end{aligned}
$$

Fig.4 shows the $C D s$ values of all experts before and after feedback when the threshold value $A C T\left(W^{1}\right)=0.917$. Fig.4(a) depicts the $C D s$ on the preference of experts before feedback. Fig.4(b) depicts the $C D s$ values of experts before and (coloured) after the feedback process, showing that experts $e_{1}, e_{2}$ and $e_{3}$ just reach the threshold value $A C T\left(W^{1}\right)$.

Obviously, different intervals of consensus threshold have different numbers of inconsistent experts. Based on the model (17), the CRP for experts with different attitudinal parameters $\alpha$ is investigated. When $0<\alpha \leq 0.363$, expert $e_{1}$ is identified as an inconsistence expert. When $0.363<\alpha \leq 0.725$, experts $e_{1}, e_{2}$ are identified as inconsistent experts. While when $0.725<\alpha \leq 1$, experts $e_{1}, e_{2}$ and $e_{3}$ are identified as inconsistent experts. The result shows that as the attitudinal parameters increase, the ACT and the number of inconsistent experts increase.

\section{Minimum adjustment cost analysis with ACT}

This section investigates the minimum adjustment cost of individual and group after feedback with different ACT. As 


$$
\begin{aligned}
& A_{\alpha=1}^{1}=\left(\begin{array}{ccc}
N_{1} & N_{2} & N_{3} \\
M_{2}\langle[0.331,0.431][0.252,0.438]\rangle & \langle[0.183,0.341][0.252,0.469]\rangle\langle[0.303,0.403][0.338,0.497]\rangle \\
M_{3}\langle[0.100,0.414][0.403,0.517]\rangle & \langle[0.169,0.386][0.310,0.528]\rangle\langle[0.300,0.600][0.200,0.300]\rangle \\
M_{4}\langle[0.245,0.534][0.269,0.500]\rangle & \langle[0.272,0.503][0.238,0.351]\rangle & \langle[0.331,0.503][0.252,0.366]\rangle
\end{array}\right)
\end{aligned}
$$

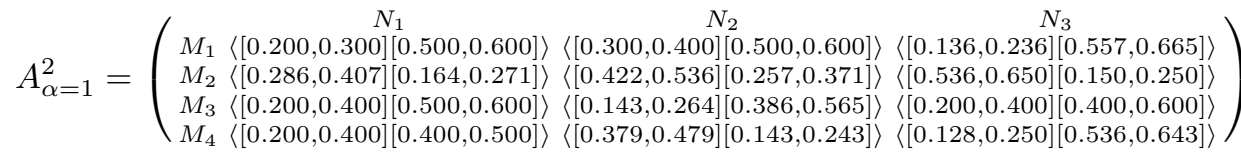

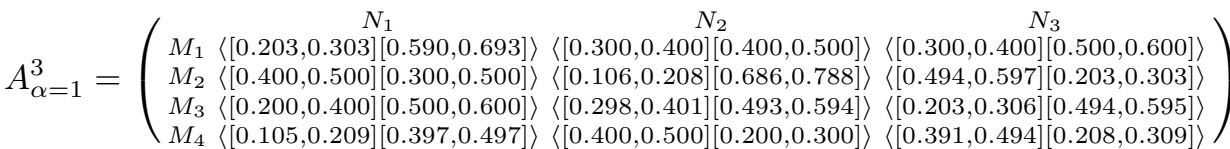

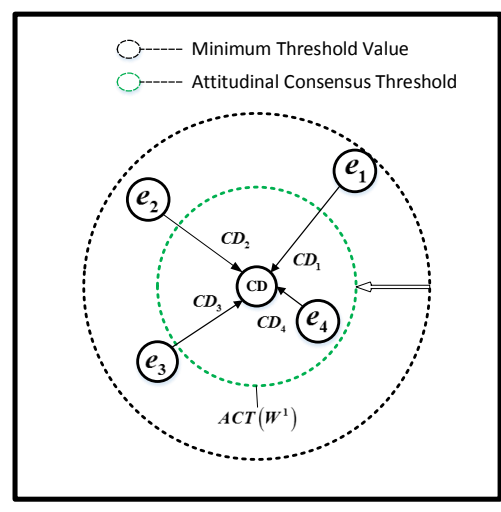

(a) Expert CDs

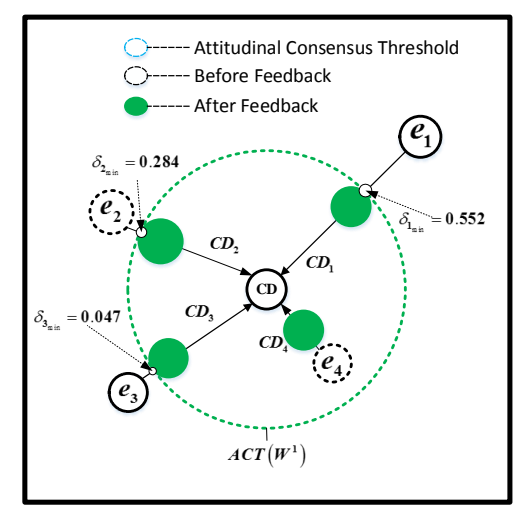

(b) CDs after feedback

Fig. 4: Consensus simulation of experts $e_{1}, e_{2}$ and $e_{3}$ by dynamic feedback mechanism.

aforementioned, inconsistent experts have different intervals of ACT. Interestingly, during the interaction between the inconsistent individual and the group, the consensus degree among other experts will also increase, which is shown in Figs (2)-(4). Therefore, the ACT interval of the feedback expert and the inconsistent expert are different. Form model (17), it can be calculated that expert $e_{1}$ generates adjustment cost when $0<\alpha \leq 1$. When $0.536<\alpha \leq 1$, expert $e_{2}$ generates adjustment cost. When $0.94<\alpha \leq 1$, expert $e_{3}$ generates adjustment cost.

The minimum adjustment cost of individual and group according to the ACT are calculated as follows:

1) The adjustment cost of expert $e_{1}$ is $T C_{1}=d_{1}^{0<\alpha \leq 1}$. $\delta_{1_{\min }}^{0<\alpha \leq 1} \in(0,3.369]$

2) The adjustment cost of expert $e_{2}$ is $T C_{2}=d_{2}^{0.516 \leq \alpha \leq 1}$. $\delta_{2_{\min }}^{0.516 \leq \alpha \leq 1} \in(0,1.271]$

3) The adjustment cost of expert $e_{3}$ is $T C_{3}=d_{3}^{0.94<\alpha \leq 1}$. $\delta_{3_{\min }}^{0.94<\alpha \leq 1} \in(0,0.17]$

4) The total adjustment cost of the group is $T C_{0<\alpha \leq 1}=$ $T C_{1}+T C_{2}+T C_{3} \in(0,4.81]$.

It is worth noting that as the ACT increases, the elements identified as inconsistency of the inconsistent experts by expression (13) will change.

$$
\text { where }\left\{\begin{array}{l}
d_{1}=\left|a_{i j}^{1}-\overline{a_{i j}}\right|, d_{2}=\left|a_{i j}^{2}-\overline{a_{i j}}\right|, \\
d_{3}=\left|a_{i j}^{3}-\overline{a_{i j}}\right| \\
d_{1}^{0<\alpha \leq 0.2}=4.45, d_{1}^{0.2<\alpha \leq 0.644}=4.95, \\
d_{1}^{0.644<\alpha \leq 0.908}=5.75, d_{1}^{0.908<\alpha \leq 1}=6.1 \\
d_{2}^{0.536<\alpha \leq 1}=4.475 \\
d_{3}^{0.94<\alpha \leq 1}=3.65
\end{array}\right.
$$

The adjustment cost of individual experts with different AC$\mathrm{T}$ intervals is shown in Table II. To visualize the influence of the expert's attitude on adjustment costs, the TC of individual experts with different ACT is shown in Fig.5, as well as the TC of the group is depicted in Fig.6. Obviously, adjustment costs of individual experts (coloured blue, orange and gray) are monotonic increasing functions with respect to the ACT in Fig.5.

In Fig.6, the result demonstrates that the CRP for inconsistent experts can be classified into three stages with different ACT: (1) if $0<\alpha \leq 0.363$, inconsistent expert $e_{1}$ reaches consensus with an adjustment cost in the interval $(0,1.507]$; (2) If $0.363<\alpha \leq 0.725$, inconsistent experts $e_{1}$ and $e_{2}$ reach consensus with an adjustment cost in the interval $(1.507,3.417]$; (3) If $0.725<\alpha \leq 1$, inconsistence experts $e_{1}, e_{2}$ and $e_{3}$ reach consensus with an adjustment cost in the interval $(3.417,4.81]$. While when $0.363<\alpha \leq 0.536$, inconsistent expert $e_{2}$ is affected by the inconsistent expert $e_{1}$ so that the group reaches consensus without adjustment cost. And when $0.725<\alpha \leq 0.94$, inconsistent expert $e_{3}$ is 
TABLE II: Adjustment cost of individual experts with different ACT intervals

\begin{tabular}{cccc}
\hline$\alpha$ & ACT interval & Feedback expert & $T C_{h}$ \\
\hline$(0,1]$ & $(0.861,0.917]$ & $e_{1}$ & $(0,3.369]$ \\
$(0.536,1]$ & $(0.894,0.917]$ & $e_{2}$ & $(0,1.271]$ \\
$(0.94,1]$ & $(0.914,0.917]$ & $e_{3}$ & $(0,0.17]$ \\
\hline
\end{tabular}

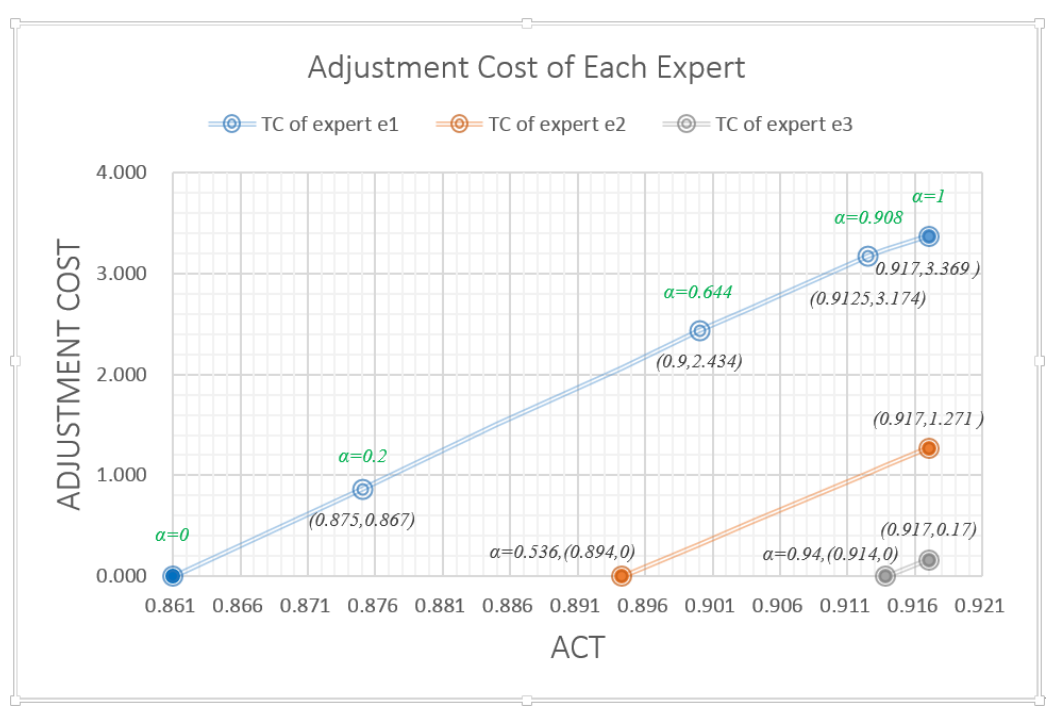

Fig. 5: Adjustment cost of individual experts after feedback with different ACT

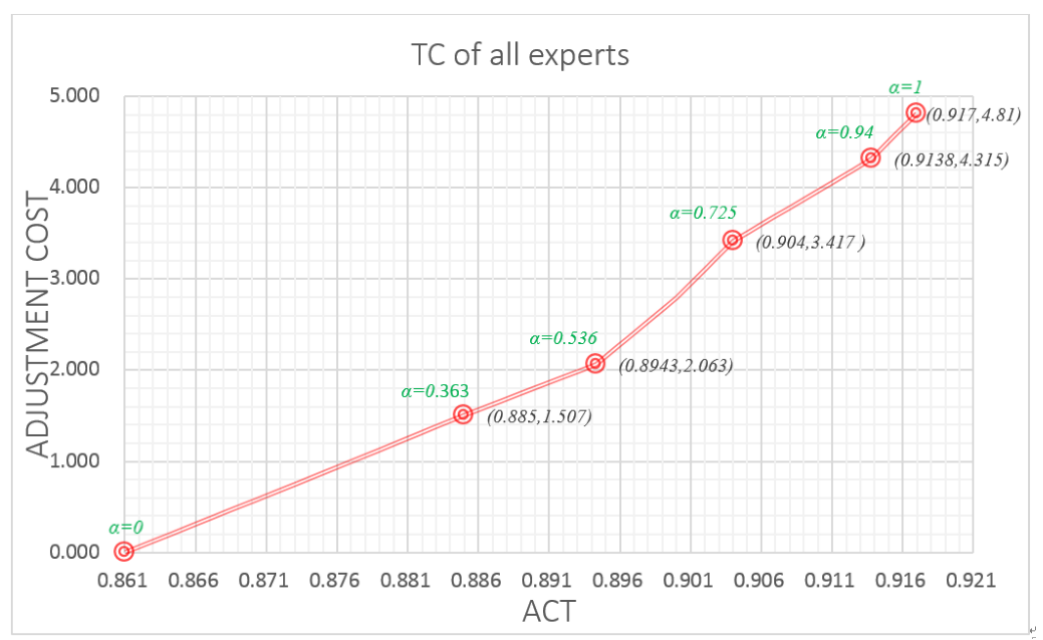

Fig. 6: Total adjustment cost of the group after feedback with different ACT

affected by the inconsistent experts $e_{1}, e_{2}$ so that the group reaches consensus without adjustment cost.

Furthermore, when the group has a high consensus requirement, i.e. $A C T(1)<\bar{\gamma}, \bar{\gamma} \in(0.917,1]$, from model (18), we can get the model (22). By solving it, the CDs of all experts after feedback process reach the new threshold value of consensus: $\bar{\gamma}=0.972$ with the total adjustment cost of $T C=18.21$. Obviously, in this case, the adjustment cost increases rapidly for reaching consensus due to the higher consistency requirements. This result is consistent with the actual GDM situation, and it supposes that the proposed dynamic feedback mechanism is reasonable in determining the adjustment cost according to different attitude. The total adjustment costs of the group for reaching consensus with different threshold of consensus degree are shown in Table III. In such table, $\frac{\Delta(T C)}{\Delta(C D)}$ represents the approximate slope of the ratio of cost to consensus threshold under different number of inconsistent experts. The results show that when the consensus threshold is within the interval $\left[C D_{\min }, C D_{\max }\right]$, the adjustment cost increases with respect to the threshold of consensus 
at a relatively slow rate, while when the consensus threshold exceeds $C D_{\max }$, reaching consensus requires relatively large adjustment costs. This will lead to more waste of resources.

$$
\begin{aligned}
& \operatorname{Min} T C=\sum_{i, j \in A P S} \sum_{h=1}^{4} \delta_{\sigma(h)} \cdot\left|a_{i j}^{\sigma(h)}-\overline{a_{i j}}\right| \\
& \text { s.t. }\left\{\begin{array}{l}
\vartheta_{i j}^{\sigma(4)}=\left(1-\delta_{\sigma(4)}\right) \cdot a_{i j}^{\sigma(4)}+\delta_{\sigma(4)} \cdot \overline{a_{i j}} \\
\vartheta_{i j}^{\sigma(3)}=\left(1-\delta_{\sigma(3)}\right) \cdot a_{i j}^{\sigma(3)}+\delta_{\sigma(3)} \cdot \overline{a_{i j}} \\
\vartheta_{i j}^{\sigma(2)}=\left(1-\delta_{\sigma(2)}\right) \cdot a_{i j}^{\sigma(2)}+\delta_{\sigma(2)} \cdot \overline{a_{i j}} \\
\vartheta_{i j}^{\sigma(1)}=\left(1-\delta_{\sigma(1)}\right) \cdot a_{i j}^{\sigma(1)}+\delta_{\sigma(1)} \cdot \overline{a_{i j}} \\
\overline{a_{i j}}=\frac{1}{4} \times \sum_{h=1} \\
C D^{\sigma(4)}<\gamma, \overline{a_{i j}^{\sigma(h)}} \\
C D^{\sigma(3)}<\gamma, \overline{C D^{\sigma(4)}}=\gamma \\
C D^{\sigma(2)}<\gamma, \overline{C D^{\sigma(2)}}=\gamma \\
C D^{\sigma(1)}<\gamma, \overline{C D^{\sigma(1)}}=\gamma \\
0.917<\gamma \leq 1 \\
\delta_{\sigma(1)} \leq \delta_{\sigma(2)}, \delta_{\sigma(2)} \leq \delta_{\sigma(3)}, \delta_{\sigma(3)} \leq \delta_{\sigma(4)} \\
0 \leq \delta_{\sigma(1)}, \delta_{\sigma(2)}, \delta_{\sigma(3)}, \delta_{\sigma(4)} \leq 1
\end{array}\right.
\end{aligned}
$$

\section{E. Ranking order of alternatives}

Without loss of generality, it is assumed that the group adopts an attitudinal parameter $\alpha=0.6$ in the decision scenario with the following attribute weights of criteria: $\omega=$ $\left(N_{1}=0.6 ; N_{2}=0.1 ; N_{3}=0.3\right)^{T}$. After feedback process we get the following overall preference value of the four alternatives $\left\{M_{1}, M_{2}, M_{3}, M_{4}\right\}$ shown in $\bar{A}_{\alpha=0.6}$ :

Using the score function of an IVIFN $S(\widetilde{\alpha})=$ $\frac{\mu^{-}\left(2-\mu^{+}-\nu^{+}\right)+\mu^{+}\left(2-\mu^{-}-\nu^{-}\right)}{2}$ in [56], the following score values are determined:

$$
M_{1}=0.362 ; M_{2}=0.480 ; M_{3}=0.385 ; M_{4}=0.394
$$

Therefore, we get the final consensus ranking: $M_{2} \succ M_{4} \succ$ $M_{3} \succ M_{1}$.

\section{F. Discussion}

Traditional consensus measurement usually requires a completely unanimous agreement, which contains only two states of 0 (absence of or partial consensus) and 1 (complete consensus). However, in real life, completely unanimous consensus is very difficult to achieve. Kacprzyk [57] proposed the concept of "soft" consensus measurement. Later, many researchers have carried out researches on "soft" consensus, which usually aims to achieve a consensus threshold in the interval $[0.5,1)$ ([18]-[27]).

(1) On the one hand, the above research works do not consider how the consensus threshold affect the adjustment cost, and then do not explain how to select an appropriated consensus threshold. In our proposed method, the consensus threshold is fixed in the interval $\left[C D_{\min }, C D_{\max }\right]$, which can intuitively help the group observe the change of the adjustment cost with different ACT intervals. The results show that the adjustment cost to reach a consensus within ACT interval increases slowly and linearly, as shown in Table III. Moreover, we obtain $\frac{T C_{\alpha=1}-T C_{\alpha=0}}{C D_{\max }-C D_{\min }}=85.89$.

(2) On the other hand, when the consensus threshold is greater than $C D_{\max }$, the adjustment cost required to reach consensus will increase rapidly $\left(\frac{\Delta(T C)}{\Delta(C D)}=243.64 \gg 85.89\right)$, which will consume a lot of adjustment cost. So, in this case, reaching consensus among the experts in the group is obviously unreasonable and unacceptable. Therefore, it may be unpractical to the actual decision-making problem that the threshold is set to exceed $C D_{\max }$.

Therefore, the main purpose of ACT proposed in this article is to aid inconsistent experts adjusting their preferences, within the reasonable scope, to reach consensus and keep their original preference as much as possible based on the different consistency requirements of the group so as to ensure the rationality of decision-making results.

\section{CONCLUSiON}

This article proposes a dynamic feedback mechanism based on attitudinal consensus threshold (ACT) for GDM problems with different consistency requirements. To achieve this, the concepts of consensus degree (CD) between individual experts and the group and the attitude-OWA operator are used to compute both the aggregation weighting vector and the group ACT. The ACT based minimum adjustment cost feedback mechanism is investigated for the inconsistent experts to reach consensus with minimum adjustment cost. The proposed dynamic feedback mechanism for GDM problems has the following main advantages and differences in comparison with other consensus models introduced in the literature:

(i) The ACT is introduced by taking into account the attitude of the group, leading to a dynamic consensus threshold in a continuous state from $C D_{\min }$ to $C D_{\max }$, avoiding the static property of the existing feedback mechanisms based on the use of the same fixed threshold for all inconsistent experts. Therefore, our proposed dynamic feedback mechanism has the flexibility to deal with GDM problems with different consistency requirements.

(ii) It builds an ACT based dynamic minimum adjustment cost feedback mechanism to generate personalised advice for the inconsistent experts based on their boundary feedback parameters to reach the ACT. This in turn makes possible for the inconsistent experts to achieve a balance between their individual independence, by modifying their original assessment the minimum possible, and the collective aim of reaching consensus.

(iii) It proposes a sensitivity analysis method with regard to the ACT which shows that the number of feedback experts monotonic increases with the ACT interval. Additionally, the adjustment cost is a monotonic increasing function with respect to the ACT parameter $\alpha$. Indeed, the greater the attitudinal parameter is, the greater the adjustment costs are. In addition, the adjustment costs will increase fast when the consensus requirement exceeds the maximum threshold. 
TABLE III: Total adjustment cost of reaching with different threshold of consensus degree

\begin{tabular}{ccccc}
\hline$\alpha$ & CTI & inconsistent expert for consensus & TC & $\frac{\Delta(T C)}{\Delta(C D)}$ \\
\hline$(0,0.363]$ & $(0.861,0.885]$ & $e_{1}$ & $(0,1.507]$ & 62.79 \\
$(0.363,0.725]$ & $(0.885,0.904]$ & $e_{1}, e_{2}$ & $(1.507,3.417]$ & 100.53 \\
$(0.725,1]$ & $(0.904,0.917]$ & $e_{1}, e_{2}, e_{3}$ & $(3.417,4.81]$ & 107.15 \\
- & $(0.917,0.972]$ & $e_{1}, e_{2}, e_{3}, e_{4}$ & $(4.81,18.21]$ & 243.64 \\
\hline
\end{tabular}

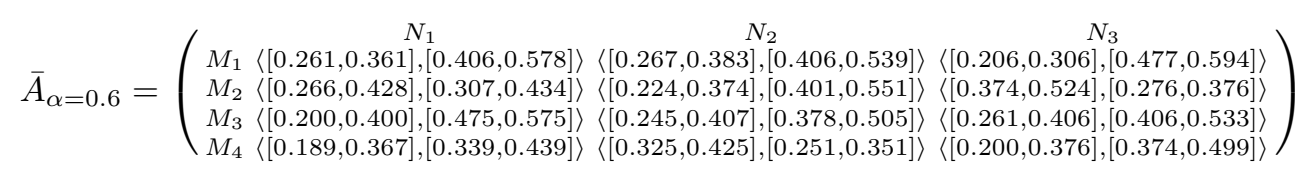

The consensus model researched in this paper has been applied on a small-scale GDM problem. In future, we will apply this dynamic method to large-scale group decisionmaking to solve real-life problems within a social network framework. Meanwhile, negotiation between individuals and groups is an interesting research direction. Specifically, in the feedback process the inconsistent individual experts makes some adjustments but fails to reach a consensus, meanwhile he/she may reluctant to make adjustments again. So, the group may be required to makes adjustments in the directing reaching a consensus. We will conduct in-depth research with regard to this issue in the future.

\section{ACKNOWLEDGEMENTS}

The authors would like to thank the editors and anonymous referees for their careful work and valuable comments and suggestions that have helped improve this paper substantially. This work was supported by National Natural Science Foundation of China (NSFC) (No.71971135, 71571166, 72071056 and 71910107002), Innovative Talent Training Project of Graduate Students in Shanghai Maritime University of China (No.2019YBR017) and was supported in part by the Spanish State Research Agency under Project PID2019-103880RBI00/AEI/10.13039/501100011033.

\section{REFERENCES}

[1] Y. J. Xu, F. J. Cabrerizo, and E. Herrera-Viedma, "A consensus model for hesitant fuzzy preference relations and its application in water allocation management," Applied Soft Computing, vol. 58, pp. 265-284, 2017.

[2] E. Herrera-Viedma, F. Chiclana, F. Herrera, and S. Alonso, "Group decision-making model with incomplete fuzzy preference relations based on additive consistency," IEEE transactions on systems, man, and cybernetics. Part B, Cybernetics : a publication of the IEEE Systems, Man, and Cybernetics Society, vol. 37, pp. 176-89, 2007.

[3] Y. J. Xu, X. W. Wen, and W. C. Zhang, "A two-stage consensus method for large-scale multi-attribute group decision making with an application to earthquake shelter selection," Computers \& Industrial Engineering, vol. 116, pp. 113-129, 2018.

[4] H. J. Zhang, Y. C. Dong, and E. Herrera-Viedma, "Consensus building for the heterogeneous large-scale gdm with the individual concerns and satisfactions," IEEE Transactions on Fuzzy Systems, vol. 26, pp. 884898, 2018.

[5] A. Rothstein and C. L. Butler, "On conflict and consensus: A handbook on formal consensus decision making," Takoma Park, 2006.

[6] Z. Zhang, X. Y. Kou, W. Y. Yu, and C. H. Guo, "On priority weights and consistency for incomplete hesitant fuzzy preference relations," Knowledge-Based Systems, vol. 143, pp. 115-126, 2018.

[7] Z. Zhang, K. X. Yue, and D. Q. Xing, "Additive consistency analysis and improvement for hesitant fuzzy preference relations," Expert Systems with Applications, vol. 98, pp. 118-128, 2018.
[8] Y. J. Xu, Q. Q. Wang, F. J. Cabrerizo, and E. Herrera-Viedma, "Methods to improve the ordinal and multiplicative consistency for reciprocal preference relations," Applied Soft Computing, vol. 67, pp. 479-493, 2018.

[9] Z. W. Gong, N. Zhang, and F. Chiclana, "The optimization ordering model for intuitionistic fuzzy preference relations with utility functions," Knowledge-Based Systems, vol. 162, pp. 174-184, 2018.

[10] Z. B. Wu, S. Huang, and J. P. Xu, "Multi-stage optimization models for individual consistency and group consensus with preference relations," European Journal on Operational Research, vol. 275, pp. 182-194, 2019.

[11] C. C. Li, Y. Gao, and Y. C. Dong, "Managing Ignorance Elements and Personalized Individual Semantics Under Incomplete Linguistic Distribution Context in Group Decision Making,"Group Decision and Negotiation, 2020, DOI:10.1007/s10726-020-09708-9.

[12] X. X. Zhang, B. F. Ge, J. Jiang, and Y. J. Tan, "Consensus building in group decision making based on multiplicative consistency with incomplete reciprocal preference relations," Knowledge-Based Systems, vol. 106, pp. 96-104, 2016.

[13] Y. C. Dong, Q. B. Zha, H. J. Zhang, G. Kou, H. Fujita, F. Chiclana, and E. Herrera-Viedma, "Consensus reaching in social network group decision making: Research paradigms and challenges," KnowledgeBased Systems, vol. 162, pp. 3-13, 2018.

[14] Q. X. Dong, X. Zhou, and L. Martínez, "A hybrid group decision making framework for achieving agreed solutions based on stable opinions," Information Sciences, vol. 490, pp. 227-243, 2019.

[15] T. Wu, X. W. Liu, J. D. Qin, and F. Herrera, "Consensus evolution networks: A consensus reaching tool for managing consensus thresholds in group decision making," Information Fusion, vol. 52, pp. 375-388, 2019.

[16] Y. C. Dong, J. Xiao, H. J. Zhang, and T. Wang, "Managing consensus and weights in iterative multiple-attribute group decision making," Applied Soft Computing, vol. 48, pp. 80-90, 2016.

[17] L. Martínez and J. Montero, "Challenges for improving consensus reaching process in collective decisions," New Mathematics and Natural Computation, vol. 50, pp. 231-250, 2019.

[18] F. J. Cabrerizo, F. Chiclana, I. Javier Perez, F. Mata, and S. Alonso, "A feedback mechanism based on granular computing to improve consensus in gdm," Soft Computing Applications for Group Decision-making and Consensus Modeling, vol. 357, 2018.

[19] M. S. Cao, J. Wu, F. Chiclana, R. Ureña, and E. HerreraViedma, "A personalized feedback mechanism based on maximum harmony degree for consensus in group decision making," IEEE Transactions on Systems, Man and Cybernetics: Systems, 2019, DOI:10.1109/TSMC.2019.2960052.

[20] J. Wu, F. Chiclana, and E. Herrera-Viedma, "Trust based consensus model for social network in an incomplete linguistic information context," Applied Soft Computing, vol. 35, pp. 827-839, 2015.

[21] J. Wu, F. Chiclana, H. Fujita, and E. Herrera-Viedma, "A visual interaction consensus model for social network group decision making with trust propagation," Knowledge-Based Systems, vol. 122, pp. 39-50, 2017.

[22] J. Wu, Z. W. Zhao, Q. Sun, and H. Fujita,"A maximum self-esteem degree based feedback mechanism for group consensus reaching with the distributed linguistic trust propagation in social network," Information Fusion, 2020, doi.org/10.1016/j.inffus.2020.10.010.

[23] Q. X. Dong, K. Y. Zhü, and O. Cooper, "Gaining consensus in a moderated group: A model with a twofold feedback mechanism," Expert Systems with Applications, vol. 71, pp. 87-97, 2017. 
[24] Z. B. Wu, B. M. Jin, and J. P. Xu, "Local feedback strategy for consensus building with probability-hesitant fuzzy preference relations," Applied Soft Computing, vol. 67, pp. 691-705, 2018.

[25] J. Wu, J. L. Chang, Q. W. Cao, and C. Y. Liang, "A trust propagation and collaborative filtering based method for incomplete information in social network group decision making with type-2 linguistic trust," Computers \& Industrial Engineering, vol. 127, pp. 853-864, 2019.

[26] J. Wu, M. S. Cao, F. Chiclana, and E. Herrera-Viedma, "An optimal feedback model to prevent manipulation behaviour in consensus under social network group decision making," IEEE Transactions on Fuzzy Systems, 2020, DOI:10.1109/TFUZZ.2020.2985331.

[27] Y. C. Dong, H. J. Zhang, and E. Herrera-Viedma, "Consensus reaching model in the complex and dynamic magdm problem," Knowledge-Based Systems, vol. 106, pp. 206-219, 2016.

[28] R. R. Yager and N. Alajlan, "An intelligent interactive approach to group aggregation of subjective probabilities," Knowledge-Based Systems, vol. 83, pp. 170-175, 2015.

[29] I. Palomares, R. M. Rodríguez, and L. Martínez, "An attitude-driven web consensus support system for heterogeneous group decision making," Expert Systems with Applications, vol. 50, pp. 139-149, 2013.

[30] J. Wu, X. Li, F. Chiclana, and R. R. Yager, "An attitudinal trust recommendation mechanism to balance consensus and harmony in group decision making," IEEE Transactions on Fuzzy Systems, vol. 27, pp. 2163-2175, 2019.

[31] J. Wu, Q. Sun, H. Fujita, and F. Chiclana, "An attitudinal consensus degree to control the feedback mechanism in group decision making with different adjustment cost," Knowledge-Based Systems, vol. 164, pp. 265-273, 2019.

[32] G. F. Yu, W. Fei, and D. F. Li, "A compromise-typed variable weight decision method for hybrid multi-attribute decision making," IEEE Transactions on Fuzzy Systems, vol. 27, pp. 861-872, 2019.

[33] J. Wu, L. F. Dai, H. Fujita, F. Chiclana, and E. Herrera-Viedma, "A minimum adjustment cost feedback mechanism based consensus model for group decision making under social network with distributed linguistic trust," Information Fusion, vol. 41, pp. 232-242, 2018.

[34] Z. Zhang and C. H. Guo, "Notes on 'logarithmic least squares method to priority for group decision making with incomplete fuzzy preference relations'," Applied Mathematical Modelling, vol. 40, pp. 1788-1792, 2016.

[35] J. F. Chu, X. W. Liu, Y. M. Wang, and K.-S. Chin, "A group decision making model considering both the additive consistency and group consensus of intuitionistic fuzzy preference relations," Computers \& Industrial Engineering, vol. 101, pp. 227-242, 2016.

[36] H. Fujita, A. Gaeta, V. Loia and F. Orciuoli "Hypotheses Analysis and Assessment in counter-terrorism activities: a method based on OWA and Fuzzy Probabilistic Rough Sets," IEEE Transactions on Fuzzy Systems, vol. 28, pp. 831-845, 2020.

[37] K. Atanassov and G. Gargov, "Interval-valued intuitionistic fuzzy sets," Fuzzy Sets and Systems, vol. 31, pp. 343-349, 1989.

[38] E. Herrera-Viedma, F. J. Cabrerizob, J. Kacprzykc, and W. Pedrycz, "A review of soft consensus models in a fuzzy environment," Information Fusion, vol. 17, pp. 4-13, 2014.

[39] Z. S. Xu, "A method based on distance measure for interval-valued intuitionistic fuzzy group decision making," Information Sciences, vol. 180, pp. 181-190, 2010.

[40] X. L. Zhang and Z. S. Xu, "Soft computing based on maximizing consensus and fuzzy topsis approach to interval-valued intuitionistic fuzzy group decision making," Applied Soft Computing, vol. 26, pp. $42-56,2015$.

[41] M. M. Deza and E. Deza, "Encyclopedia of distances," Springer, Berlin Heidelberg, 2009.

[42] J. Wu, F. Chiclana, and H. C. Liao, "Isomorphic multiplicative transitivity for intuitionistic and interval-valued fuzzy preference relations and its application in deriving their priority vectors," IEEE Transactions on Fuzzy Systems, vol. 26, pp. 193-202, 2018.

[43] Y. J. Liu, C. Y. Liang, F. Chiclana, and J. Wu, "A trust induced recommendation mechanism for reaching consensus in group decision making," Knowledge-Based Systems, vol. 119, pp. 221-231, 2017.

[44] E. Herrera-Viedma, M. Francisco, L. Martínez, and F. Chiclana, "A consensus support system model for group decision-making problems with multigranular linguistic preference relations," IEEE Transactions on Fuzzy Systems, vol. 13, pp. 644-658, 2005.

[45] R. R. Yager, "Quantifier guided aggregation using OWA operators," International Journal of Intelligent Systems, vol. 11, pp. 49-73, 1996.

[46] M. O'Hagan, "Aggregating template or rule antecedents in real-time expert systems with fuzzy set logic," Asilomar Conference on Signals. IEEE Computer Society, 1988.
[47] X. W. Liu, "On the properties of equidifferent rim quantifier with generating function," International Journal of General Systems, vol. 34, pp. 579-594, 2005.

[48] X. W. Liu and L. H. Chen, "On the properties of parametric geometric owa operator," International Journal of Approximate Reasoning, vol. 35, pp. 163-178, 2004.

[49] X. W. Liu and Q. L. Da, "On the properties of regular increasing monotone (rim) quantifiers with maximum entropy," International Journal of General Systems, vol. 37, pp. 167-179, 2008.

[50] D. Ben-Arieh and T. Easton, "Multi-criteria group consensus under linear cost opinion elasticity," Decision Support Systems, vol. 43, pp. 713-72, 2007.

[51] N. Zhang, Z. W. Gong, and F. Chiclana, "Minimum cost consensus models based on random opinions," Expert Systems with Applications, vol. 89, pp. 149-159, 2017.

[52] Y. Li, H. J. Zhang, and Y. C. Dong, "The interactive consensus reaching process with the minimum and uncertain cost in group decision making," Applied Soft Computing, vol. 60, pp. 202-212, 2017.

[53] B. W. Zhang, Y. C. Dong, and Y. F. Xu, "Multiple attribute consensus rules with minimum adjustments to support consensus reaching," Knowledge-Based Systems, vol. 67, pp. 35-48, 2014.

[54] Y. T. Liu, Y. C. Dong, H. M. Liang, F. Chiclana, and E. HerreraViedma, "Multiple attribute strategic weight manipulation with minimum cost in a group decision making context with interval attribute weights information," IEEE Transactions on Systems, Man, and Cybernetics: Systems, vol. 49, pp. 1981-1992, 2019.

[55] X. Tian, Z. W. Gong, F. Chiclana, and N. Zhang, "Consensus modeling with cost chance constraint under uncertainty opinions," Applied Soft Computing, vol. 67, pp. 721-727, 2018.

[56] Z. S. Xu and Q. L. Da, "An overview of operators for aggregating information," International Journal of Intelligent Systems, vol. 18, pp. 953-969, 2010.

[57] J. Kacprzyk, "On some fuzzy cores and 'soft' consensus measures in group decision making," In: Bezdek, J.C. (Ed) The Analysis of Fuzzy Information, pp. 119-130, CRC Press, Boca Raton, 1987.

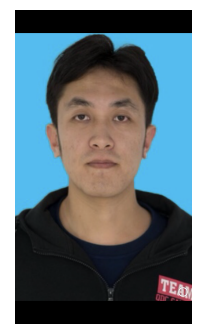

Qi Sun received the B.Sc. degree in Information Management and Information System from Shenyang Aerospace University, Shenyang, China, in 2017 and the M.Sc. degree in Management Science and Engineering from Shanghai Maritime University, Shanghai, China, in 2019. He is currently enrolled in a Ph.D. degree at Shanghai Maritime University. His research interest are mainly in group consensus process and social network. His publication is in Knowledge-Based Systems.

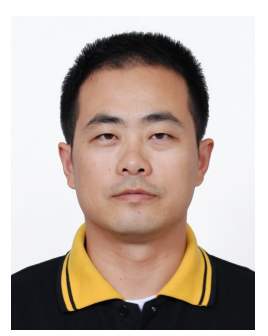

Jian Wu (SM'16) received the B.Sc. and Ph.D degrees in Management Science and Engineering from Hefei University of Technology, Hefei, China, in 2000 and 2008, respectively. He is a Distinguished Professor with the School of Economics and Management, Shanghai Maritime University, Shanghai, China. From October 2012 to October 2013, he was an Academic Research Visitor with the Centre for Computational Intelligence, De Montfort University, Leicester, U.K. He has 60+ papers published in leading journals such IEEE Transactions on Fuzzy Systems, IEEE Transactions on Systems, Man, and Cybernetics:Systems, Information Fusion, Information Sciences, Knowledge-Based Systems, Expert Systems with Applications, Applied Soft Computing. Fourteen papers have been classed as Highly Cited Papers by the Essential Science Indicators, six of them are HOT paper. One of his research papers was awarded the prestigious Emerald Citations of Excellence for 2017. His research interests include group decision making, social network, fuzzy preference modeling, and information fusion.

Prof. Wu is an Area Editor of the Journal of Computers \& Industrial Engineering, Associate Editor of the Journal of Intelligent and Fuzzy Systems and a Guest Editor of Applied Soft Computing. 


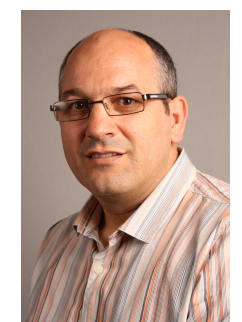

Francisco Chiclana received the $\mathrm{BSc}$ and $\mathrm{PhD}$ in Mathematics from the University of Granada, Spain, in 1989 and 2000, respectively. He is a Professor of Computational Intelligence and Decision Making with the School of Computer Science and Informatics, Faculty of Computing, Engineering and Media, De Montfort University, Leicester, U.K. He is an Associate Editor and a Guest Editor for several ISI indexed journals such as IEEE Transactions on Systems, Man, and Cybernetics: Systems, Information Sciences, Applied Soft Computing, and KnowledgeBased Systems. . He has organized and chaired special sessions/workshops in many major international conferences in research areas as fuzzy preference modeling, decision support systems, consensus, recommender systems, social networks, rationality/consistency, aggregation. He is currently a Highly Cited Researcher in Computer Sciences (according to Essential Science Indicators by Clarivate Analytics).

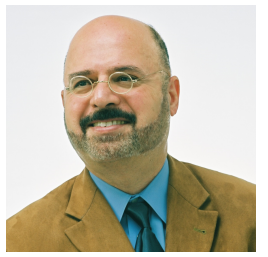

Hamido Fujita (M'82-SM'10) received the Master degree and Ph.D. degree from Tohoku University, Japan, in 1985 and 1989, respectively. He is a Distinguished Professor at Iwate Prefectural University (IPU), Iwate, Japan, as a director of Intelligent Software Systems. He received Doctor Honoris Causa from O'buda University in 2013 and also from Timisoara Technical University, Romania, in 2018, and a title of Honorary Professor from O'buda University, Budapest, Hungary, in 2011. He received honorary scholar award from University of Technology Sydney, Australia on 2012. He is Adjunct professor to Stockholm University, Sweden; University of Technology Sydney; National Taiwan Ocean University and others. He has supervised $\mathrm{PhD}$ students jointly with University of Laval, Quebec, Canada; University of Technology, Sydney, Australia; Oregon State University, Corvallis, U.S.; University of Paris-1 Pantheon-Sorbonne, France; and University of Genoa, Italy. He is a Highly Cited Researcher in Computer Sciences (according to Essential Science Indicators by Clarivate Analytics). for the year 2020., He is Emeritus Editorin-Chief of Knowledge-Based Systems. He is vice president of International Society of Applied Intelligence, and Editor in Chief of Applied Intelligence Journal (Springer). He has four international patents in software system and several research projects with Japanese industry and partners.

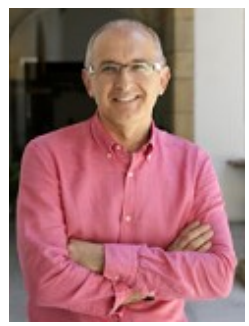

Enrique Herrera-Viedma received the M.Sc. and $\mathrm{Ph} . \mathrm{D}$ degrees in computer science from the University of Granada, Granada, Spain, in 1993 and 1996, respectively. He is a Professor of computer science and the Vice-President for Research and Knowledge Transfer with University of Granada,Granada, Spain. His h-index is 68 with more than 17000 citations received in Web of Science and 85 in Google Scholar with more than 29000 cites received. He has been identified as one of the worlds most influential researchers by the Shanghai Center and Thomson Reuters/Clarivate Analytics in both computer science and engineering in the years 2014,2015,2016,2017 and 2018. His current research interests include group decision making, consensus models, linguistic modeling, aggregation of information, information retrieval,bibliometric, digital libraries, web quality evaluation, recommender systems, and social media. Dr. Herrera-Viedma is currently Vice-President for Publications in IEEE SMC Society and an Associate Editor in several journals such as IEEE Transactions on Fuzzy Systems, IEEE Transactions on Systems, Man and Cybernetics: Systems, Information Sciences, Applied Soft Computing, Soft Computing, Fuzzy Optimization and Decision Making, and Knowledge-Based Systems. 